\title{
و اقع استخدام تطبيقات الذكاء الاصطناعي في مدارس التربية الخاصة بمدينة جدة من وجهة نظر المعلمات والاتجاه نحوها
}

\author{
ساميه فاضل الغامدي \\ باحثة دكتوراه تقنيات التعليم- جامعة الملك عبدالعزيز بجدة- المملكة العربية السعودية \\ samia.alghamdi@gmail.com$$
\text { لينا أحمد الفر اني }
$$ \\ أستاذ مساعد تقنيات التعليم- جامعة الملك عبدالعزيز بجدة- المملكة العببية السعودية
}

هدفت الدراسة إلى الكشف عن واقع استخدام معلمات التربية الخاصة للتطبيقات التعليمية للذكاء الاصطناعي والاتجاه نحوها من وجهة نظر المعلمات في معهد النور بمحافظة جدة، وقد استخدمت الدراسة المنهج الوصفي التحليلي لتحقيق أهداف الدراسة، وتكونت عينة الدراسة من (rV) معلمة من معلمات معهد النور بمحافظة جدة تم اختيارهن الطريقة القصدية من مجتمع الدراسة، وتم تصهيم استبانة كأداة لجمع المعلومات مكونة

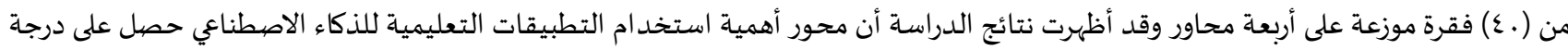
(موافق بشدة) من قبل معلمات التربية الخاصة، وحصل محور معوقات استخدام التطبيقات التعليمية للذكاء الاصطناعي، وكذلك محور الاتجاه نحو

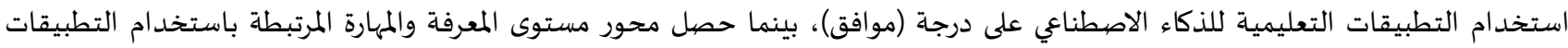
التعليمية للذكاء الاصطناعي على درجة (محايد).

الكلمات المفتاحية: التربية الخاصة؛؛ التطبيقات التعليمية؛ الذكاء الاصطناعي. (ㅇ)

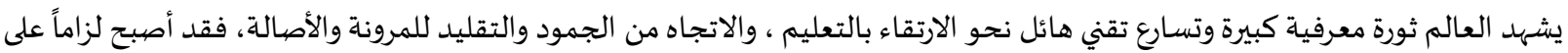
الأنظمة الدفع بقوة نحو الأخذ بعين الاعتبار النهوض بجميع مكوناتها وفي مقدهتها المعلم، وذلك انطلاقًا من كون المعلم ركيزة فهيها وأهم مدخلاتها

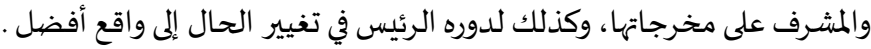

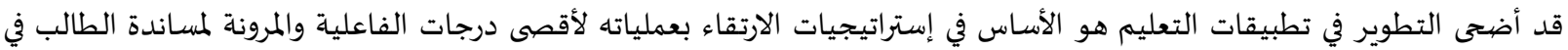

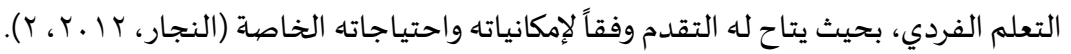

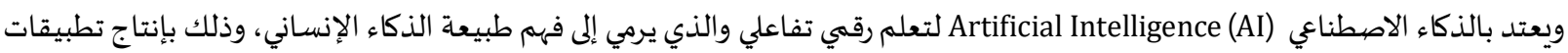
محوسبة تحاكي سلوك الإنسان الذكي، سواء بحل المسائل أو اتخاذ قرار ما، و حل المشكلات والتدريب على حلها ـ (ido\& ruth, 2016, 584) هذا ويفزو الذكاء الاصطناعي ساحة النظام المحوسب للتعلم ليتطور بواقع ملموس يمكن قياسه وذلك من خلال تقديم تقنيات ودمج وسائط

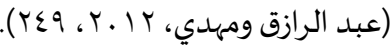
كما يوجد العديد من الاستخدامات المفيدة للذكاء الاصطناعي والتي تغطي مجالات واسعة، وأحد أهم تلك المجالات، وربما أقلها بحثاً هو

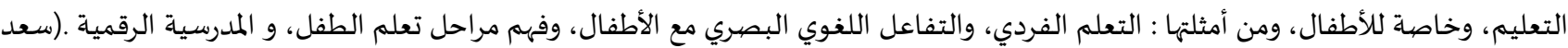


وقد بينت نتائج الدراسات السابقة التأثير الإيجابي لتوظيف التطبيقات للذكاء الاصطناعي في تعليم الطلاب ،ومن هذه الدراسات دراسة أوشانا

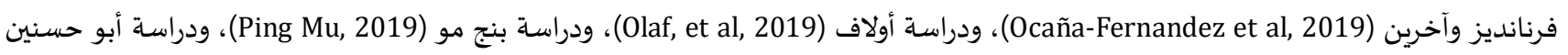

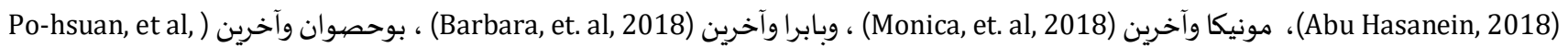

كما أن دخول الذكاء الاصطناعي للمدارس، ذو فائدة لتلاميذ الفئة الخاصة،، إذ أسهمت تكنولوجيا التعليم بـ . ^٪ )بتعلمهم ، وأزالت حواجز

حالت دون استقلالهم (Barrett \& at all, 2019) وكذلك يؤكد السيد (ع ا ـ ، إهץ) أن التقنيات الحديثة ومنها تطبيقات الذكاء الاصطناعي قد وفرت لذوي الإعاقة البصرية في المجال التعليمي ما كان خياًا في الماضي، حيث مكنتهم من استخدام الحواسيب والأجهزة اللوحية والهواتف الذكية بواسطة برامج قارئات الشاشة وأجهزة التكبير ، والتي

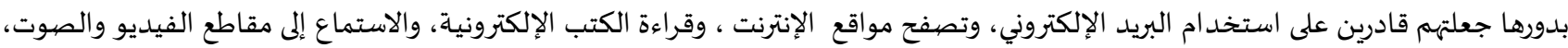
والمشاركة في مواقع التواصل الاجتماعي، وغيرها الكثير باستقلالية تامة.

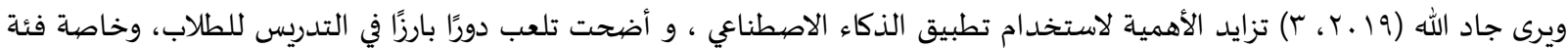

المعاقين بصرياً إذ تساعدهم على تمكينهم الاجتماعي وترفع من قدراتهم ومهاراتهم الحياتية .

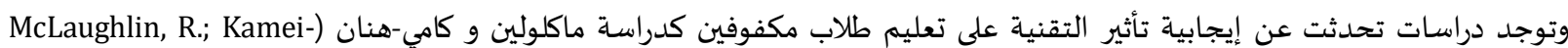

(Hannan, C., 2018 والتي استخدمت جهاز "IPAD في تعليم طلاب مكفوفين مهارات القراءة، ودراسة بيل وروزنبلوم (Beal and Rosenblum, 2018) والتي وظفت تطبيقا لجهاز لوحي "IPad" لمساعدة الطلاب المكفوفين بحل المشكلات الرياضية، ، ودراسة جو (Jo, 2015) مستخدمة الطابعة ذات الثلاثة أبعاد لطباعة خرائط تاريخ، ودراسـة لاهاف وآخرين (Lahav Et Al, 2015) تدرب المكفوفين للتنقل على بيئة افتراضية. وبناء على هذه الدراسات وغيرها أصبح من الضروري على معلمي الطلاب ذوي الإعاقة البصرية دمج التقنيات الحديثة واعتبارها عنصبرا رئيسا في كافة مراحل تدريسهم. وتسعى هذه الدراسة للبحث في واقع استخدام تطبيقات الذكاء الاصطناعي في معهد النور للكفيفات بجدة ومعوقات استخدامهن لها من وجهة نظرهن ، واتجاههن نحوها.

مشكلة الدراسـة: إن دخول ثورة تقنيات الذكاء الاصطناعي لمجال التعليم يعتبر تغييرًا كبيرًا في الأدوار التي يقوم بها كلا من المتعلم والمعلم والعاملين في وزارة

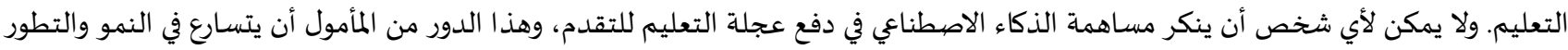
بشكل كبير، ولذا يجب مواكبة هذا التسارع المضطرد والعمل على استثمار تقنياته وتوظيفها التوظيف الأمثل من أجل تعليم أبنائنا وصلاح أوطاننا. ويعدّ موضوع تطبيقات الذكاء الاصطناعي واستخداماتها العامة وفي مجال التعليم بشكل خاص من الموضوعات الحديثة التي تعدّ مجالا خصبيا

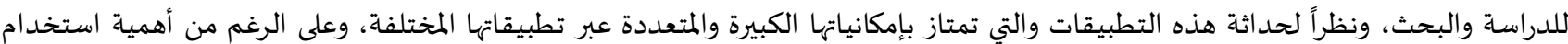

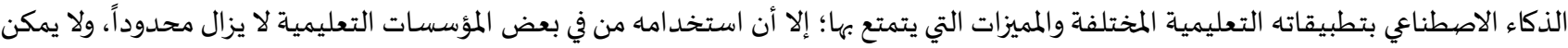

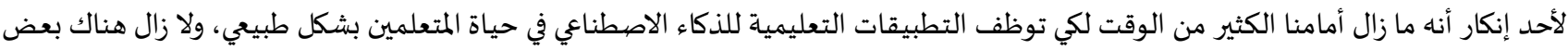

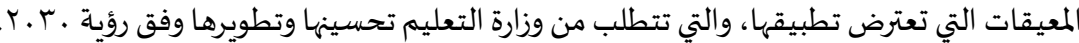

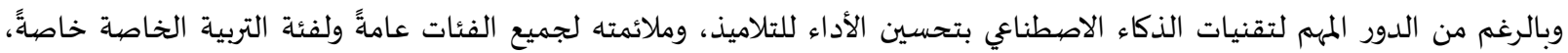

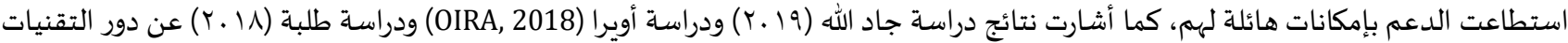
للمعاق بصريا لدعم تعلمهه إلا أن القصيور موجود في الأبحاث المتعلقة بتوظيف الذكاء الاصطناعي لهذه الفئة، لذلك نسعى في هذه الدراسة لمعرفة واقع الاستخد ام عند معاهد النور للكفيفات للتطبيقات التعليمية للذكاء الاصطناعي . ويمكن صياغة مشكلة الدراسة بسؤال رئيس هو:

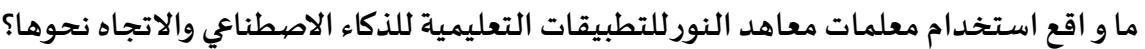
متفرعة منه الأسئلة الآتية : ا. ما مستوى معرفة ومهارة معلمات معاهد النور باستخدامات التطبيقات التعليمية للذكاء الاصطناعي؟

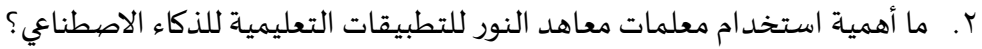

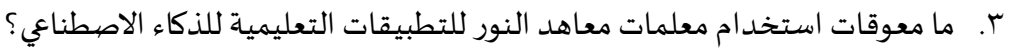
ع. ما اتجاه معلمات معاهد النور لاستخدام التطبيقات التعليمية للذكاء الاصطناعي؟ 
0. هل يوجد فروق ذات دلالة إحصائية عند (0., .) لواقع استخدامات معلمات معاهد النور للذكاء الاصطناعي والاتجاه نحوها يعزى لاستخدامهن

من وجهة نظرهن

أهداف الدراسـة: - (1)

1. تحديد مستوى استخدام معلمات معاهد النور للتطبيقات التعليمية للذكاء الاصطناعي.

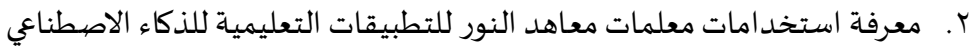

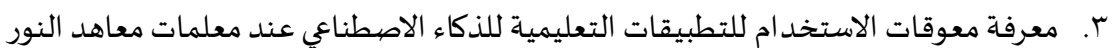
ع. معرفة الاتجاه للتطبيقات التعليمية للذكاء الاصطناعي عند معلمات معاهد النور 0. تحديد الفروق في استخدامات معلمات التربية الخاصة لتطبيقات الذكاء الاصطناعي تعزى للاستخدام من وجهة نظرهن.

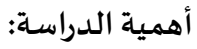
ا. تقدم هذه الدراسة بيان بمعوقات استخدام التطبيقات التعليمية للذكاء الاصطناعي لتكون بمثابة الأساس الذي يبنى عليه استراتيجيات تحسين خدمات التعلم الذكي، وتوجيه أنظار المهتمين بالعملية التعليمية وأصحاب القرار لمحاولة تذليل تلك المعوقات أمام سبل توظيف الذكاء الاصطنائي وتطبيقاته في العملية التعليمية. r. توجيه اهتمام المختصين والخبراء وصناع القرار في وزارة التربية والتعليم إلى ضرورة تبني استخدام التطبيقات التعليمية للذكاء الاصطناعي ضمن مخططاتها، الأمر الذي يسهم في تطوير استراتيجيات التدريس المستخدمة في المدارس بشكل عام ورديه

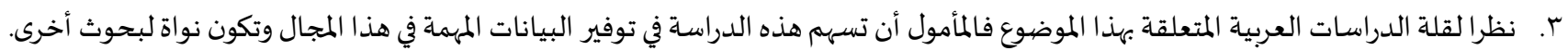
ع. قد تفيد أداة البحث الحالي باحثين آخرين على إجراء دراسات تتكامل مع نتائج البحث الحالي.

حدود الدراسـة: الدراسة مقصورة على تقديرات العينة من معلمات معاهد النور بمحافظة جدة لواقع استخد اماتهن لتطبيقات الذكاء الاصطناعي والاتجاه نحوها خلال

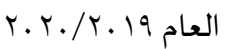

المصطلحات:

التطبيقات التعليمية لتقنيات الذكاء الاصطناعي:تعرف إجرائياً بأنها : " استخدام أجهزة أو برامج أو آلات أو أنظمة قادرة على محاكاة الذكاء البشري للقيام بعمليات ومهام محددة (مثل برامج المحادثة الفورية والروبوتات)".

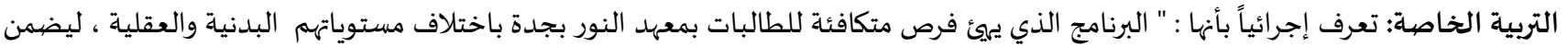

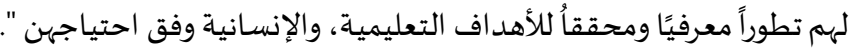

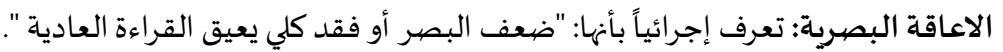
الاتجاهات: تعرف إجرائياً : اتساق استجابات القبول أو الرفض لدى معلمات معاهد النور بمحافظة جدة نحو تطبيقات تعليمية للذكاء الاصطناعي ".

\section{الإطار النظري والدراسـات السـابقة:} مفهوم الذكاء الاصطناعي:

منذ ظهور مصطلح الذكاء الاصطناعي لأول مرة في مؤتمر Dartmouth عام 1907 م ظهرت عدة تعريفات تناولت هذا المصطلح ويمكن تصنيفها

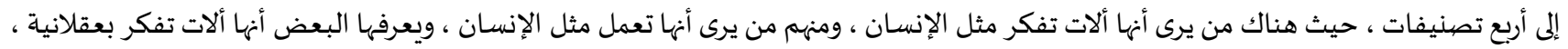

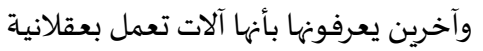
ويعرفه لطفي (1) • r) بأنه: "أحد فروع علوم الحاسوب، الذي يهتم بطرق ووسائل خلق وتصميم أجهزة وآلات ذكية تستطيع التفكير والتصرف مثل البشر والقيام بمهام متعددة تتطلب ذكاءً مثل التعلم، التخطيط، تمييز الكلام، التعرف على الوجها، حل المشاكل، الإدراك، والتفكير العقلي والمنطقي".

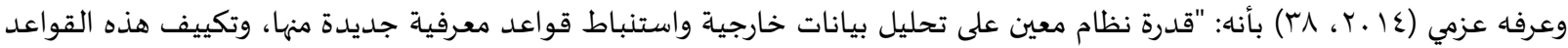

واستخد امها لتحقيق أهد اف ومهام جديدة".

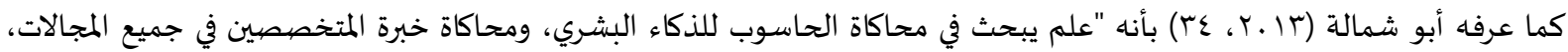
وتطوير البرامج لحل المشكلات بمعالجة البيانات والمعلومات بطرق غير خوارزمية". 
كما يعرفه (Kaplan and Haenlein, 2019,17) بأناه قدرة النظام على تفسير البيانات الخارجية بشكل صحيح ، والتعلم من هذه البيانات ، واستخد ام تلك الدروس لتحقيق اهداف ومهام محددة من خلال التكيف المرن.

تطبيقات الذكاء الاصطناعي:

يحاول الذكاء الاصطناعي استنتاج أفضل الحلول من محاولة الإنسان حل مشكلاتٍ جديدة تصادفاته في حياته اليومية، وفيما يلي بعضاً من

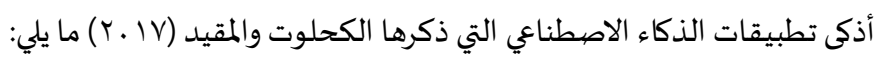

الروبوت صوفيا: أول روبوت في العالم يصبح مواطنًا شرعيًا لديها الجنسية السعودية. وتتمتِّع بصفات كالبشر ، فهي يمكنها أن تعبر عن مشاعرها،

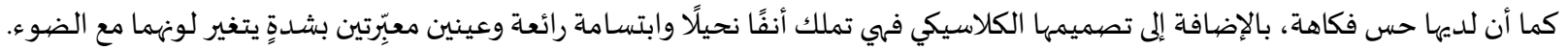
السيارات القيادة الذاتية: Tesla واحدة من أوائل العلامات التجارية للسيارات التي أطلقت مركبة تقاد ذاتيًا، تقود نفسها بدون سائق، والتي يتم

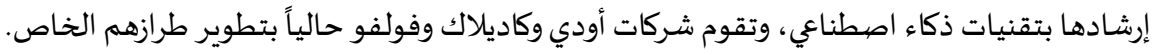

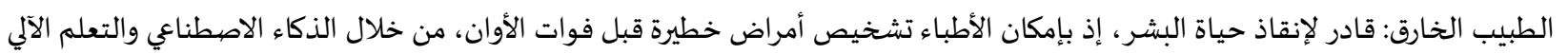
العميق، حيث يحلل الصور والوجوه لتشخيص أيّة علامات مبكّرة محتملة باستخدام الأشعة السينية. ترشيد الطاقة: حيث يوجد حاليا منازل مزوّدة بتحكّم ذو ذكاء اصطناعي قادرة على خفض استهلاك الطاقة من خلال التحكم بالمقابس الذكية وأجهزة استشعار الإضاءة الآلية . أمن المنزل: بخوارزميات تعرف ملامح الوجه، المنزل بنظام الذكاء الاصطناعي يقوم بإنشاء فهرسٍ للأفراد الّذين يعيشون فيه، وأيضًا المعروفين من

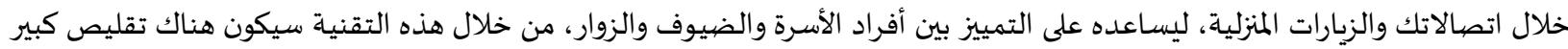

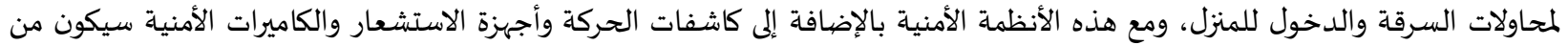
السهل تقييم الاختراق المحتمل بل وحتّى طلب خدمة الطوان الطوارئ. الحياة على كواكب أخرى: حيث قامت وكالة ناسـا أيضًا باستخدام تقنيات ذكية للبحث عن حياة على كواكب أخرى، وتتمكن الأجهزة التي يرسلونها والمعروفة باسم Rovers من استكشاف تضاريس ومكونات كوكب المريخ بتفاصيلٍ ودّقةٍ أكبر.

تطبيقات الذكاء الاصطناعي في التعليم: تمعروفي من توفر الطبيعة الرقمية والديناميكية للذكاء الاصطناعي مجالا مختلفا لا يمكن العثور عليه في البيئة التقليدية النمطية للمدرسة في وقتنا الحالي. فتطبيقات الذكاء الاصطناعي في التعليم ستمكن من اكتشاف حدود تعلم جديدة وتسرّع إنشاء تقنيات مبتكرة. ومن بين تطبيقات الذكاء

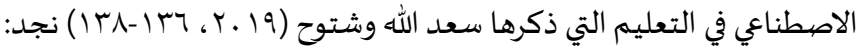

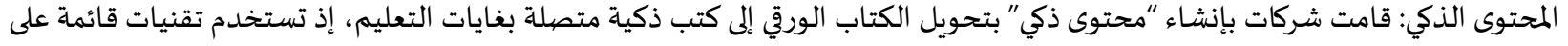
الذكاء الاصطناعي لنشرها ، وتتضمن ملخصيات الفصول واختبارات الممارسة الصحيحة والاختيارات المتعددة. كما تقوم شركات أخرى أيضاء بإنشاء منصيات محتوى ذكية متكاملة مع دمج المحتوى بتمارين الممارسة والتقييم مثل برنامج Netex Learning الذي يتيح للمعلمين تصميم

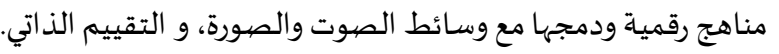

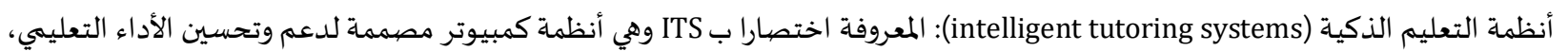
بتوفير دروس فورية دون تدخل من مدرس بشري، مستخدمة عدد من تقنيات الحوسبة والذكاء الاصطناعي. تقنيات الواقع الافتراضي (VR) والواقع المعزز(AR): تقنية (VR) هي محاكاة لخوض تجارب مختلفة كالمشاركة في مباراة لكرة القدم أو زيارة أماكن

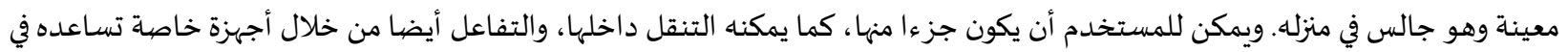

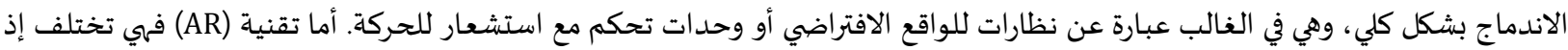

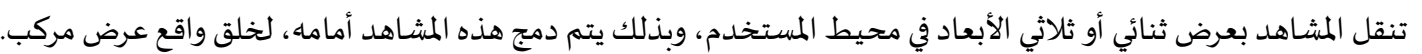
أهداف استخدامات تقنيات الذكاء الاصطناعي في التعليم: زادت الاستخدامات بالانتشار في عدد من القطاعات، ومن ذلك التعليم، بهدف أن تصل لأفضل تجربة للتعلم، ومن أبرز أهداف استخدا اماتها

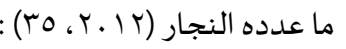
الدرجات: وفرت بعض الشركات البرامج يتم من خلالها إجراء التدريبات والاختبارات، وتحديد الدرجات، وتصحيح الإجابات، وإعلام الطلاب بأدائهم في تلك الاختبارات. 
التخصصية: وفقًا لأداء ومهارات كل طالب بعض الشركات وفرت نظام مخصص بتقييم أداء ومهارات الطلاب، وبناءً على أداء كل طالب ونقاط

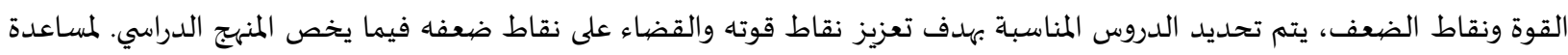
المعلمين في تحديد مستوى طلابهم بدقة ومعرفة ما يحتاجه كل طالب من المنهج حتى يمكنهاه زيادة معدلات نجاحهاه. جودة المناهج والتدريس: يستطيع الذكاء الاصطناعي تعيين الفجوات من خلال أداء الطلاب في الاختبارات والتدريبات. فمثلا، إذا قام عدد من

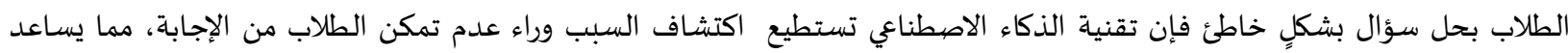
المعلمين في شرح أجزاء محددة من المنهج والتركيز علهيا بشكلٍ أفضل. بساء التدريب: وذلك في بناء مواقع وبرامج تدريب ذكية تستطيع قياس وتحديد أساليب وطرق تعلم الطلبة، وتقييم ما يمتلكونه من معرفة ثم تقديم تدرببات مخصصية وفق تقييماء. التعلم التكيفي: إحراز تقدمّات ملحوظة من خلال تعليم الطلاب بشكل فردي، كما يتم التعديل للمناهج ، وتقديم تقرير تفصيلي للمعلم عن المواد.

التقييم الفوري للطلاب: تقوم برامج التعلم بتقييم مهارات الطلاب بشكلٍ فوري ليساعد على تطور أدائهم الدراسي. التعلم عن بعد: تقدم فرصًا لتقديم الاختبار عن بُعد مع فرض أنظمة رقابية لمراقبة الطالب، والتحقق من عدم الغش، فهي طريقة يتم بها التحقق من مصد اقية ودقة الاختبار. مساعدة طلاب الاحتياجات الخاصاة: إذ تلبي احتياجاتهم وتحفيزهم على التأقلم مع الأجواء التعليمية، وزيادة مهاراتهم واستيعاب المواد الدراسية.

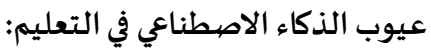

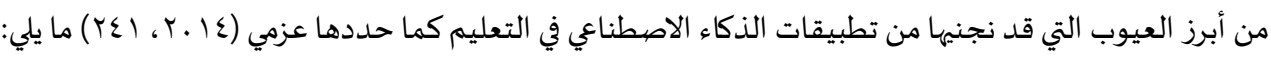
ارتفاع تكلفة تنفيذ العديد من تطبيقات الذكاء الاصطناعي في التعليم.

من المتوقع ارتفاع نسبة البطالة بين صفوف الهيئات التدريسية. احتمالية حدوث الاختراقات والنسخ الذاتي للفيروسات التي قد تغزو الروبوتات. غياب التفاعل الاجتماعي من خلال خلو الأجواء الصفية من روح التعاون والتآلف التي يقدمها المعلم للطالب. انعدام الرغبة في التعلم والشعور بالملل من جهاة الطلبة من خلال تعاملهم الدائم مع الآلة. صعوبة التعامل مع الروبوتات في غالب الأحيان. الأثار السلبية على السلوك البشري نتيجة تعامله الد ائم مع الآلات. مستقبل الذكاء الاصطناعي في التعليم: مأسئ اقترح (Woolf, 2013) مجموعة من التحديات الكبيرة التي ينبني أن يعمل الذكاء الاصطناعي على معالجتها، بما في ذلك: معلمّ افتراضي لكل متعلم: حيث يكون هناك دعمٌ كلي يجمع بين نمذجة المستخدم والمحاكاة الاجتماعية وتمثيل المعرفة. مواكبة مهارات القرن الحادي والعشرين: مساعدة المتعلمين في التوجياه والتقييم الذاتي والعمل الجماعي وما إلى ذلك. تححليل بيانات التفاعل: تجميع كميات هائلة من البيانات حول التعلم الفردي والسياقات الاجتماعية وسياقات التعلم والاهتمامات الشخصياة. توفير الفرص للفصول الدراسية العالمية: زيادة الترابط وسهولة الوصول إلى الفصول الدراسية في جميع أنحاء العالم. تقنيات مدى الحياة: أخذ التعلم خارج الفصل الدراسي والدخول إلى حياة المتعلم خارج المدرسـة.

وبالاطلاع على عدد من الدراسات السابقة عن الذكاء الاصطناعي مثل: دراسـة أوشانا فرنانديز وآخرين (Ocaña-Fernandez et al, 2019) والتي هدفت إلى التعرف على الذكاء الاصطناعي وانعكاساته في التعليم

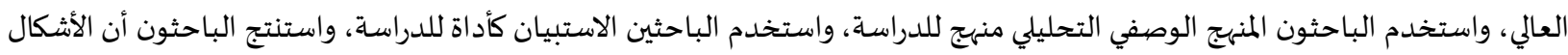
المستندة إلى الذكاء الاصطناعي تؤدي إلى تحسن كبير في التعليم لكافة المستويات التعليمياة، مع تحسين نوعي غير مسبوق، مع تزويد الطلاب

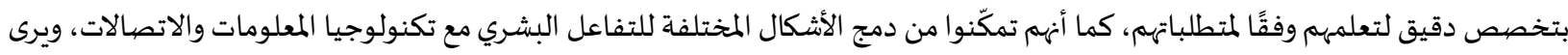
الباحثون أن التحدي الأكبر الذي تواجهه الجامعة في الألفية الجديدة يكمن في الحاجة الماسـة إلى تخطيط المهارات المهات الرقمية وتصهميمها وتطويرها

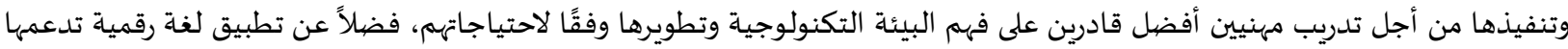
برامج الذكاء الاصطناعي. 
دراسة أبو حسنين (Abu Hasanein, 2018) وهدفت الدراسـة إلى تصميم مدرس خصوصي ذكي باستخدام أداة (ITSB) لتطوير عملية تدريس مساق مهارات الحاسوب وهو مساق مقر كمتطلب جامعة يدرساه طلاب جامعة الإسراء على عينات مختلفة من الطلاب، واستخدم الباحث المهج

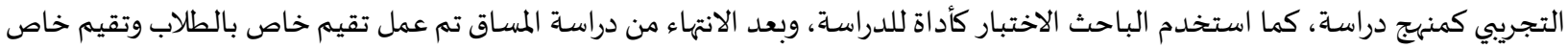

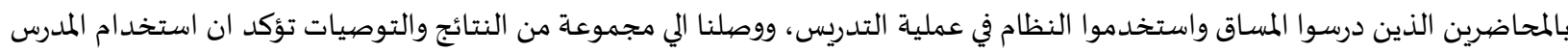

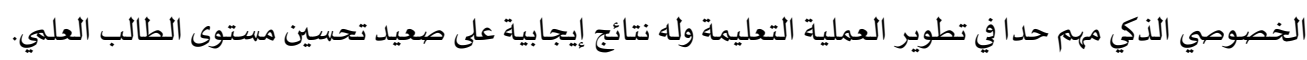

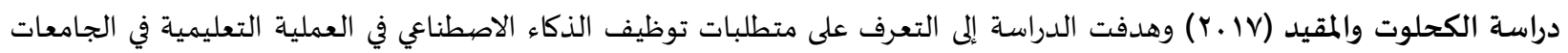

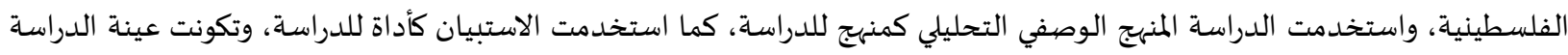

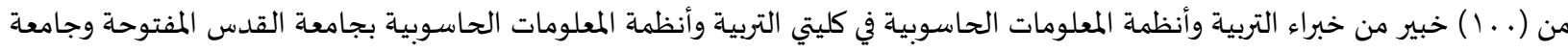

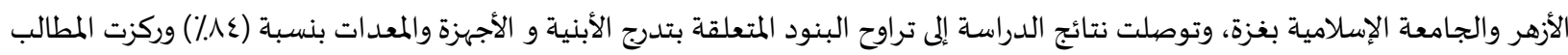

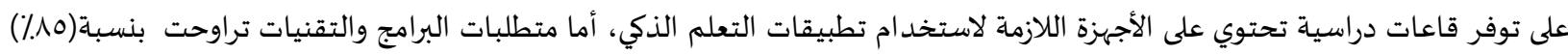

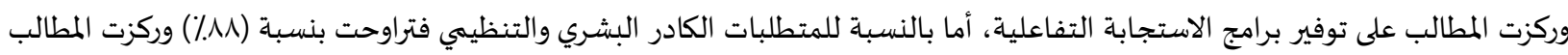

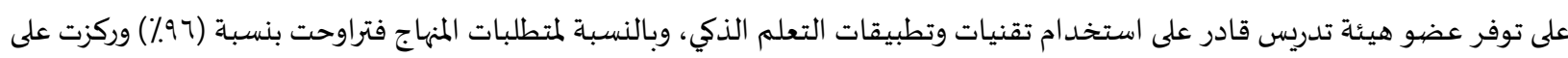
تنوع أساليب التقويم.

دراسة آل سعود (7) ـ ب) وهدفت الدراسة إلى التعريف بمفهوم الذكاء الاصطناعي، ونشأته وظهوره، والفرق بينه وبين الذكاء الإنساني، إضيافة

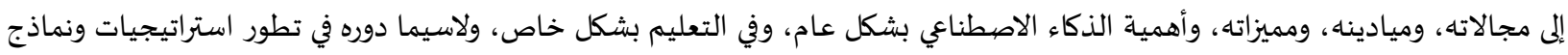

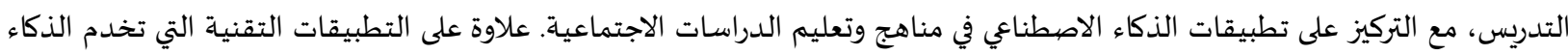

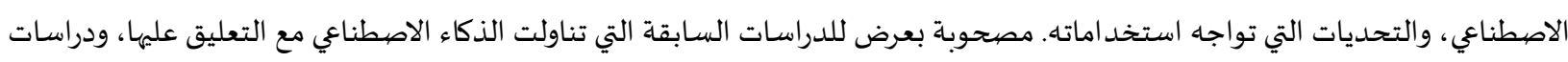
مقترحة في مجال الدراسات الاجتماعياة. دراسة عزمي (عا ـr) والتي هدفت إلى التعرف على فاعلية بيئة تعلم إلكترونية قائمة على الذكاء الاصطناعي لحل مشكلات صيانة شبكات الحاسب لدى طلاب تكنولوجيا التعليم، واستخدمت الدراسة المنهج شبه التجريبي من خلال استخد ام اختبار تحصيلي لقياس الجانب المعادي المعرفي في

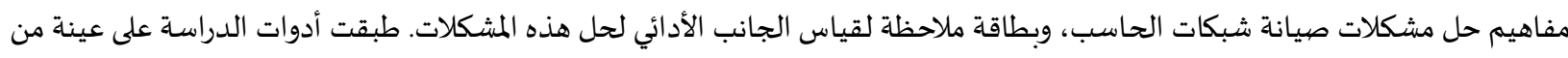

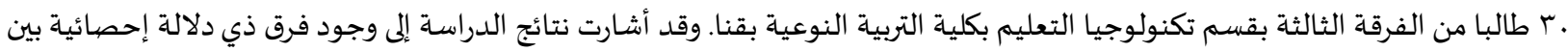

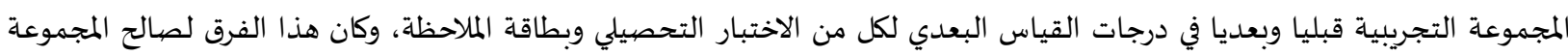

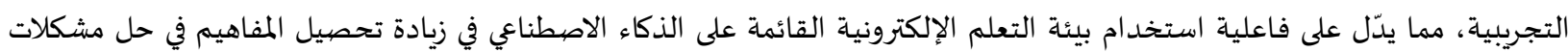
صيانة شبكات الحاسب لدى طلاب شعبة تكنولوجيا التعليم بكلية التربية النوعية. دراسة أبو شمالة (با ـ Y) وهدفت الدراسة إلى معرفة فاعلية برنامج قائم على الذكاء الاصطناعي لتنمية التفكير الاستدلالي والتحصيل الدراسي

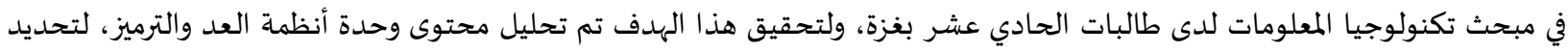

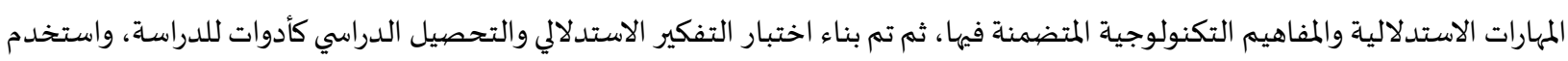

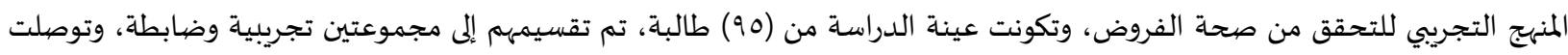

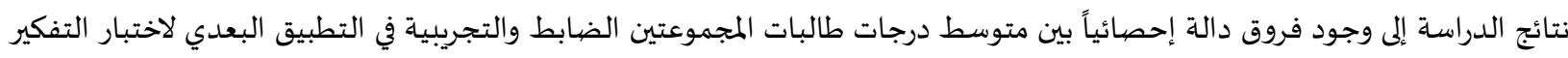

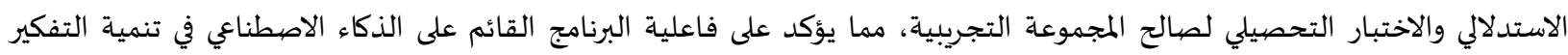
الاستدلالي والتحصيل الدراسي.

دراسة الجيار(با ـ r) والتي هدفت إلى التعرف على توظيف الذكاء الاصطناعي لبناء مواقع الإنترنت التعليمية كمدخل لتطوير التعليم الجامعي

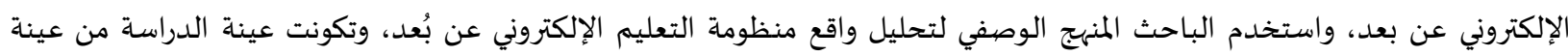

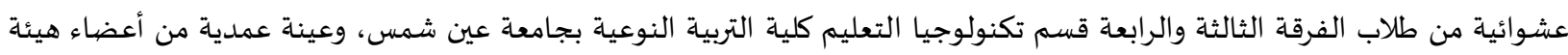

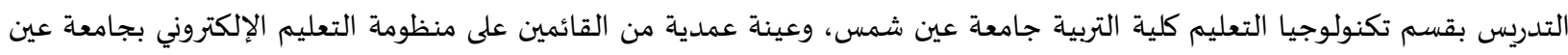

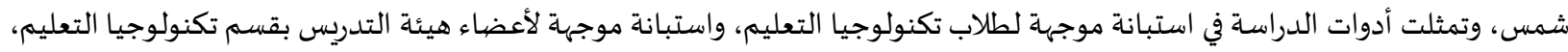
واستبانة موجهة للقائمين على منظومة التعليم الإلكتروني بجامعة عين شمس، وقائمة معايير الجودة لنظم التعليم الإلكتروني الذكية عبر

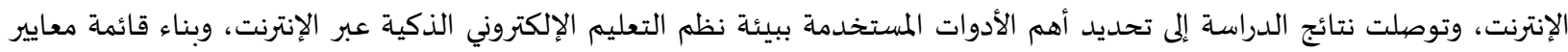

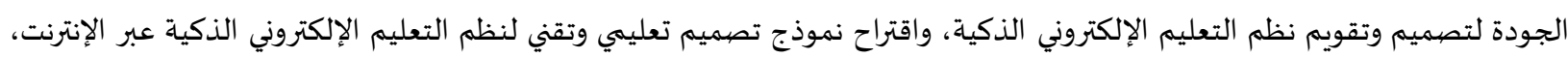

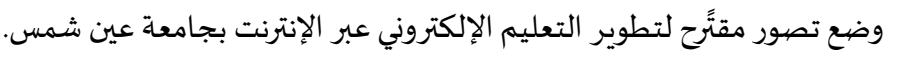


ومن الدراسات السابقة التي تناولت توظيف التكنولوجيا في تعليم الأشخاص ذوي الإعاقة البصرية:

دراسة جاد الله (19 •r) والتي هدفت إلى التعرف على فاعلية برنامج تدريبي قائم على تطبيقات جوجل التعليمية في تنمية مهارات التعلم

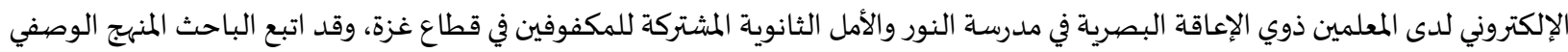
في تحديد قائمة مهارات التعلم الإلكتروني المراد تنميتها لدى المعلمين ذوي الإعاقة البصرية، وبناء البرنامج التدريبي وفق نموذج (VIVID) للتصيميم

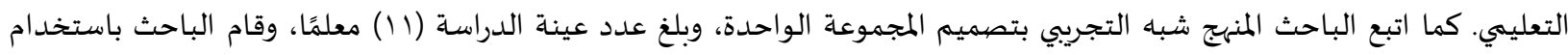
ببطاقة ملاحظة كأداة للدراسة، وقد أظهرت نتائج الدراسة وجود فروق ذات دلالة إحصائية بين متوسطي درجات المعلمين ذوي الإعاقة البصرية في التطبيق القبلي والبعدي على بطاقة الملاحظة المتعلقة بمهارات التعلم الإلكتروني لصالح التطبيق البعدي، كما أظهرت النتائج اتصاف التهاف البرنامج التدريبي بالفاعلية في تنمية مهارات التعلم الإلكتروني لدى المعلمين ذوي الإعاقة البصرية. دراسة العتوم وآخرين (1) ـ r) وهدفت الدراسة إلى الكشف عن دور التقنيات المساندة الخاصة بالمكفوفين في تمكينهم الاجتماعي. واستخدم

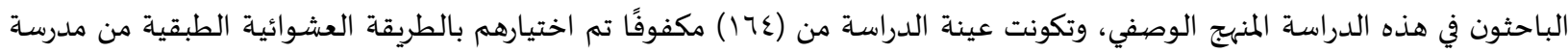
الأسقفية الكاثوليكية في مدينة إببد ونادي الشعلة في عمان، وتمثلت أدوات الدراسة باستبانة استخدمت لتقدير تمكينهم الاجتماعي، وأظهرت

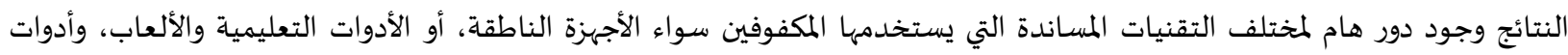

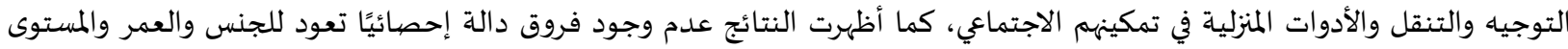

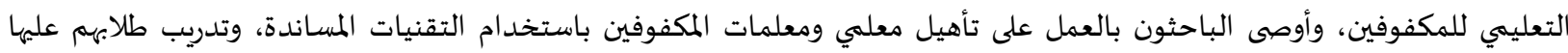
وتزويدهم بها بغض النظر عن مناطقهم.

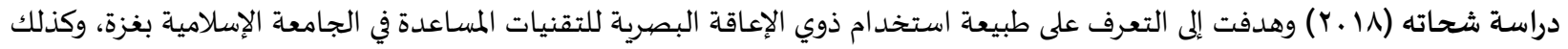

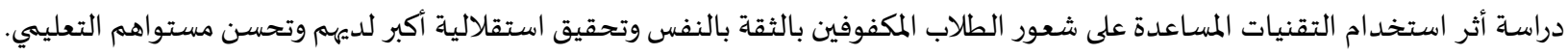
وقد استخدم الباحث في إجراء الدراسة المنهج الوصفي التحليلي ودراسة الحالة، واستخدم أداتين لجمع البيانات الأولية، وهما: المقابلات

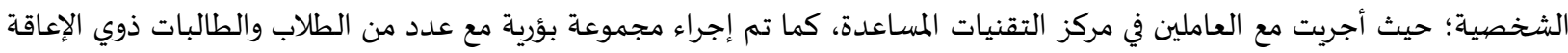

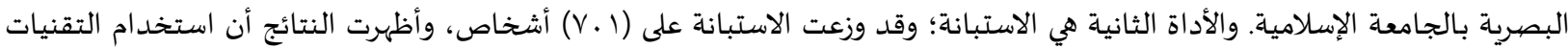

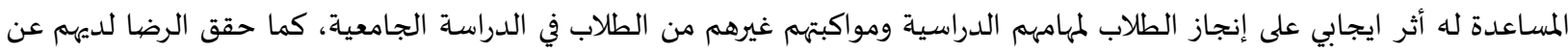

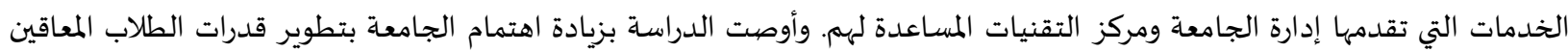

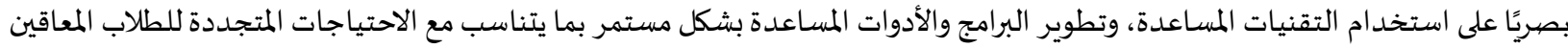

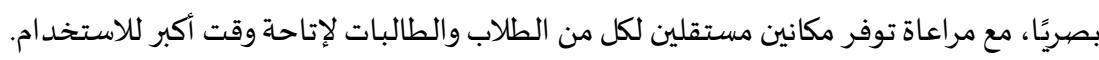

دراسة طلبة (1) إY) وهدفت إلى الكشف عن أثر استخدام البرمجيات التعليمية مفتوحة المصدر OER في تنمية الكفايات الإلكترونية الأدائية

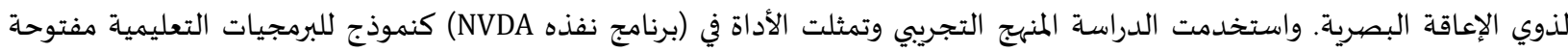

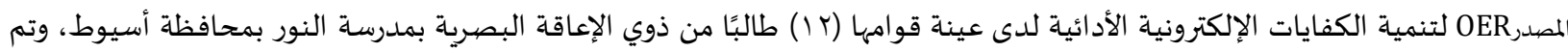

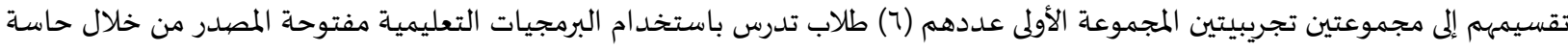

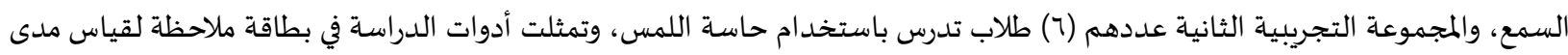
تمكنهم من الجانب الأدائي للكفايات الإلكترونية، وقد خلصتئ نتائج الدراسة إلى أنه توجد فروق ذات دلالة إحصائية بين متوسطي درجات عينة

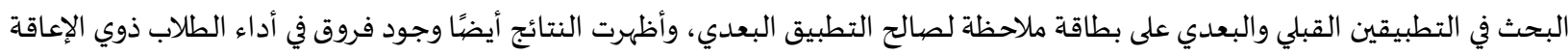
البصرية قبل وبعد استخدام برنامج نفده لصالح التطبيق البعدي، وأظهرت النتائج فاعلية البرمجيات التعليمية مفتوحة المصددر باستخدام

حاسة السمع.

دراسة مايكوي (MAKOYE, 2018) وهدفت إلى الكشف عن التحديات التي تواجه الطلاب ذوي الإعاقة البصرية في الوصول إلى التعليم الإلكتروني في مدرسة تابورا الثانوية للبنات في تنزانيا، كما سعت الدراسة إلى التعرف على المشكلات التي تواجه الطلاب المعاقين بصريا في الوصول

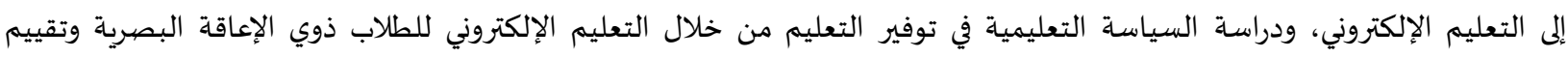

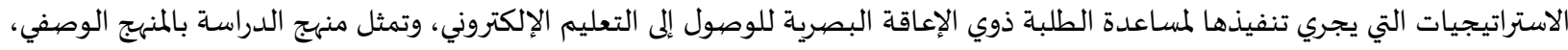
وتكونت عينة الدراسة من (.0) من الطلاب ذوي الإعاقة البصرية والمعلمين وموظفي الموارد. وقد تم جمع البيانات باستخدام الاستبيانات المنظمة والمقابلات. وقد كشفت نتائج الدراسة أن هناك العديد من المشاكل الأكاديمية التي تواجه الطلاب ذوي الإعاقة بصريًا في الوصول إلى في إلى

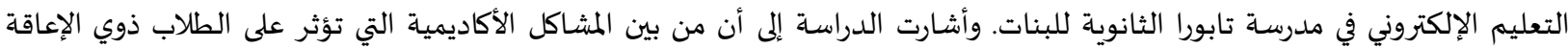


البصرية، عدم كفاية المعدات مثل: (المسجل الرقهي، وبطاقة الذاكرة، وطريقة "بريل"، وجهاز قارئ الكتب الإلكترونية، ومرافق تكنولوجيا المعلومات والاتصالات بما في ذلك: أجهزة الكمبيوتر - وأجهزة التكبير - وقارئات الشاشة) الماشة). دراسة الشرمان وآخرين (Y (Y) وهدفت إلى محاولة فهم واقع الأشخاص المكفوفين في المجتمع الأردني من حيث قدرتهم على التكيف والاندماج في مختلف النشاطات الاجتماعية، ومحاولة الكشف عن دور التقنيات التكيفية الحديثة الخاصة بالمكفوفين في تفعيل هذا التمكين والدميج المجتمعي، وذلك من وجهة نظر المكفوفين أنفسهم، واتبعت الدراسة المنهج الوصفي التحليلي، وتكونت عينه الدراسة من عينة قصدية تكاية تكونت من

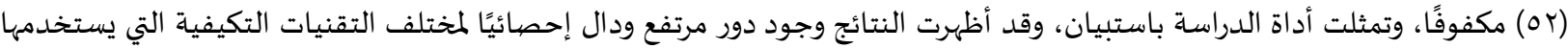

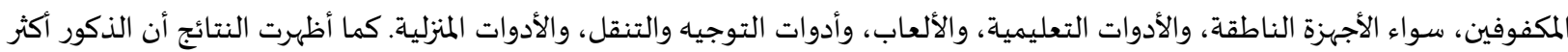

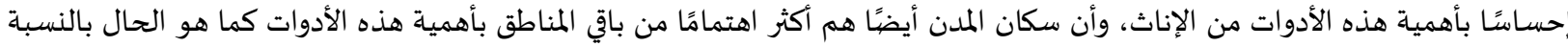

لفئة الحاصلين على شهادات عليا.

دراسة مصطفى (Mostafa, 2017) وهدفت إلى التعرف على أثر تصميمين مختلفين من برامج قارئات الشاشة في تنمية مهارات استخدام

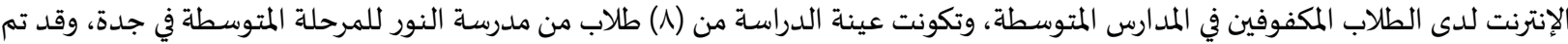

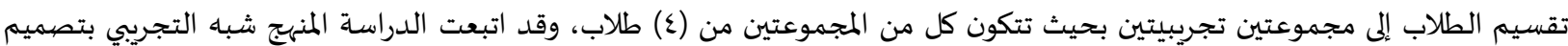

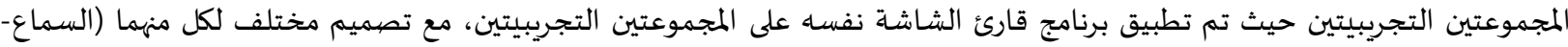
اللمس). وقد تمثلت أداة الدراسة ببطاقة ملاحظة، وأظهرت نتائج الدراسة أنها توجد فروق ذات المات دلالة إحصائية بين متوسطي درجات المجموعتين التجريبتين في الأداء المهاري لصالح المجموعة التجريبية التي درست بتصميم قارئ الشاشة المعتمد على السماع.

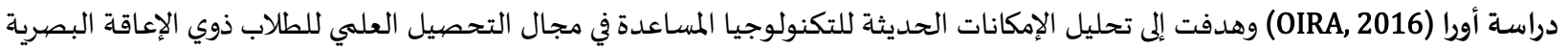

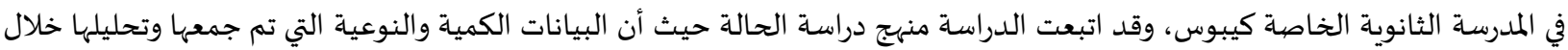

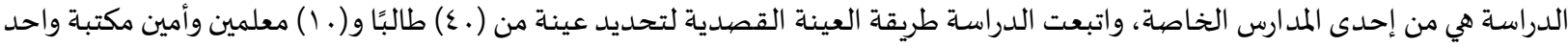

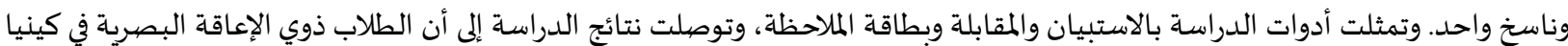

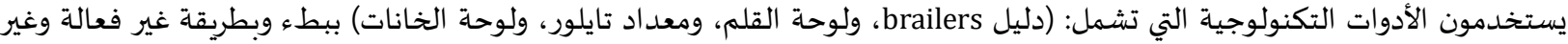

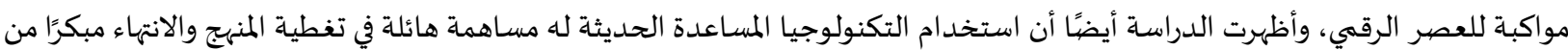

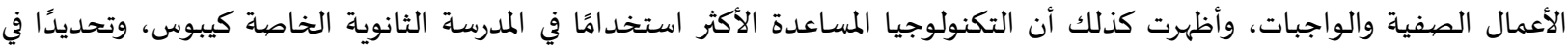

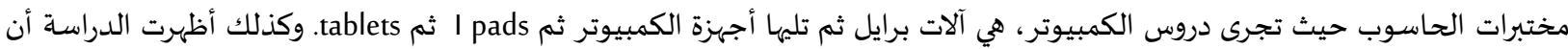

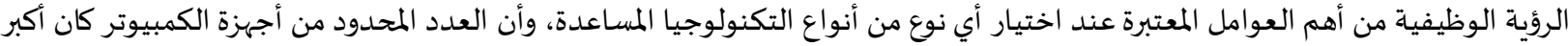

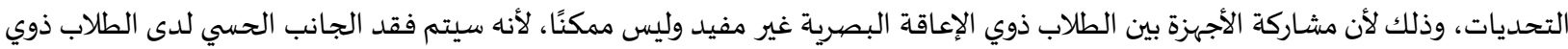

دراسة المغاربة والحميدان (17. (Y) وهدفت إلى التعرف على الاحتياجات التدرببية لمعلمي الطلبة ذوي الإعاقة البصرية في محافظتي عمان

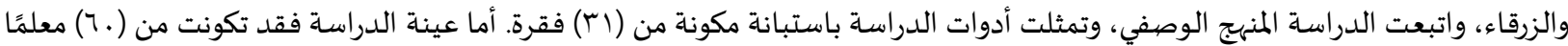

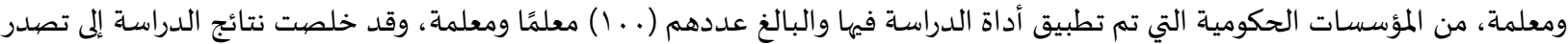

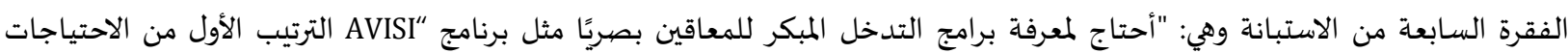
التدريبية من وجهة نظر معلمي الطلبة ذوي الإعاقة البصرية، بينما احتلت الفقرة العاشرة من الاستبانة وهي: "أحتاج التدرب على القائل القراءة

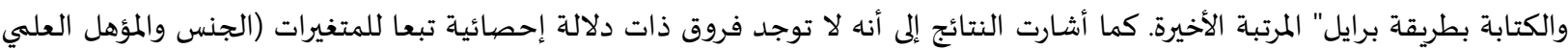

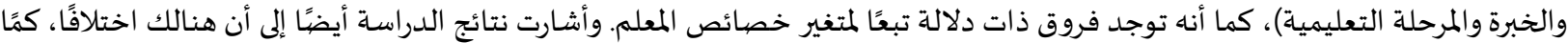

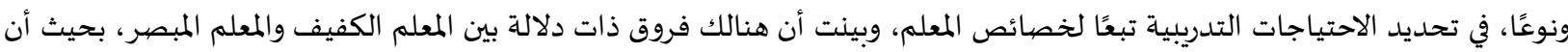

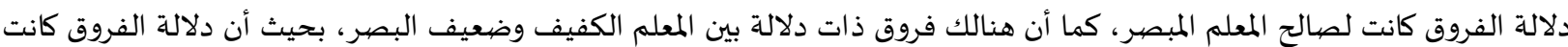
لصالح المعلم ضعيف البصر.

دراسة زيدان (10 ـ ب) وهدفت إلى الكشف عن أثر استخدام برنامجي NVDA JAWS على تنمية التحصيل لدى الطالبات ذوي الإعاقة البصرية

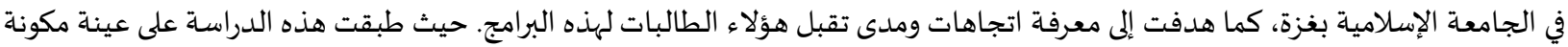

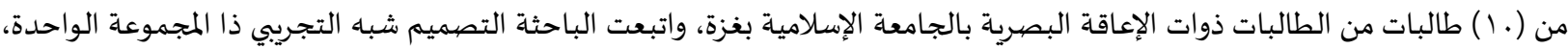

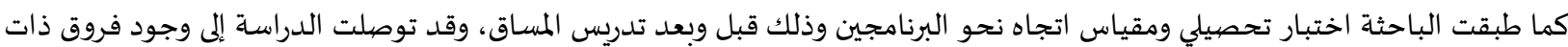
دلالة إحصائية بين التطبيق القبلي والبعدي في اختبار التحصيل التابع لبرنامج JAWS وذلك لصالح التطبيق البعدي حيث أظهر تحسن في في التبالي 
مستوى أداء أفراد العينة التجريبية في التطبيق البعدي، وكذلك وجود فروق ذات دلالة إحصائية بين التطبيقين القبلي والبعدي في مقياس

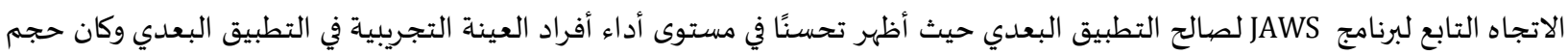

الأثر كبيرًا جداً.

التعقيب على الدراسات السابقة:

تتفق العديد من الدراسات السابقة الواردة في هذه الدراسة مع الدراسة الحالية في استخدام المنهنج الوصيفي التحليلي ومن هذه الدراساسات

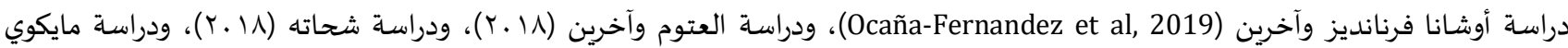

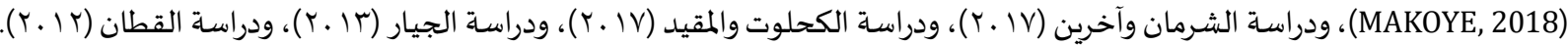

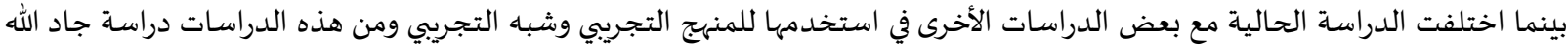

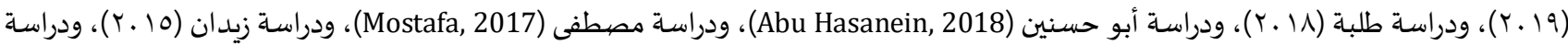

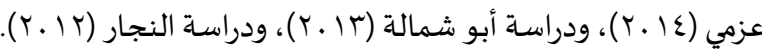

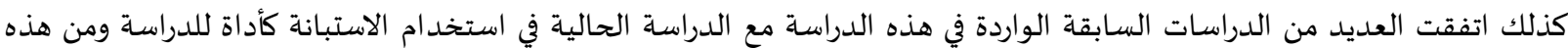

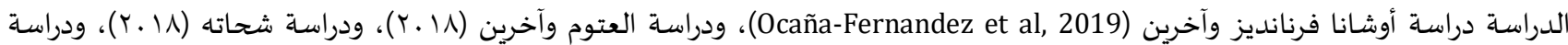

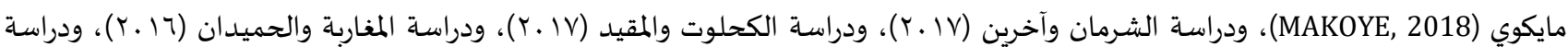

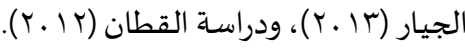

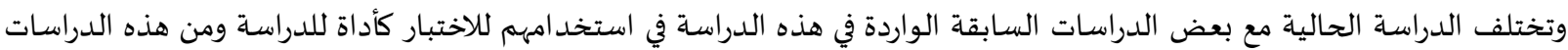

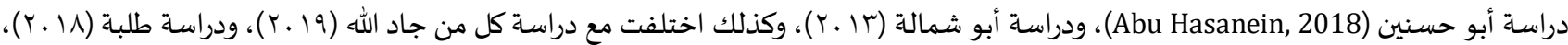
ودراسة مصطفى (Mostafa, 2017) في استخدامهم لبطاقة الملاحظة كأداة للدراسة. بينما استخدمت بعض تلك الدراسات أكثر من أداة مثل دراسة أورا (OIRA, 2016) والتي استخدمت الاستبانة وبطاقة ملاحظة كأدوات

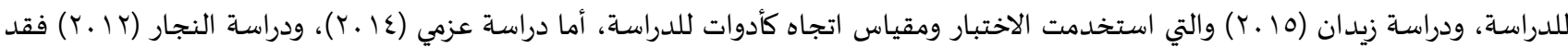
استخدمت الاختبار وبطاقة ملاحظة كأدوات للدراسة.

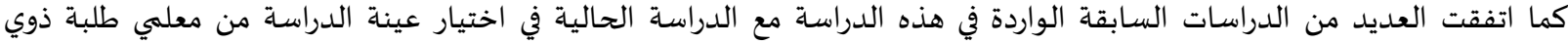

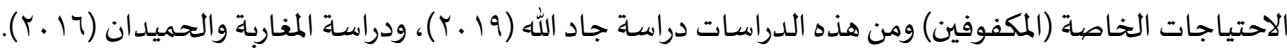

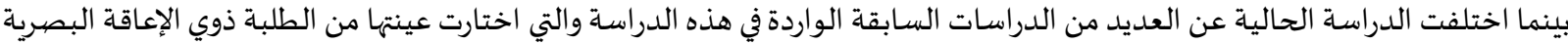

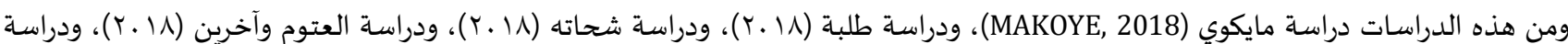

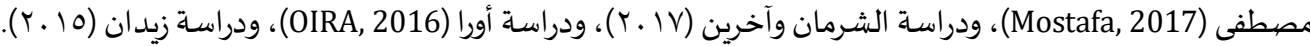

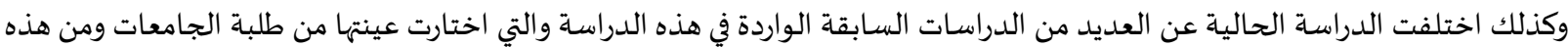

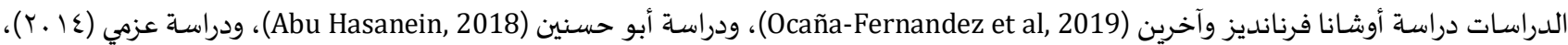

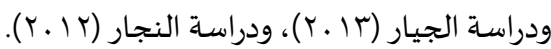

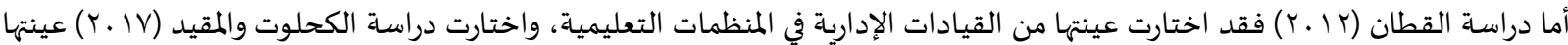
من أعضاء هيئة التدريس بالجامعات. الإجراءات:

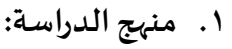
بالاعتماد على التحليل الوصفي، بهدف تحديد وضع قائم لظاهرة، ونقاط قوتها وضعفها، من أجل معرفة مدى صلاحيتها، والخروج بتعميمات

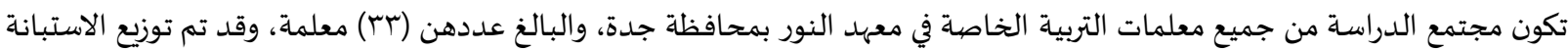

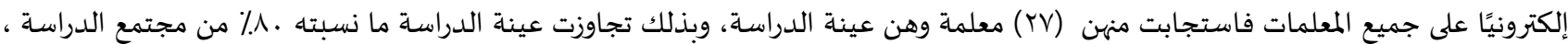
وجدول (1) يبين توزيع أفراد عينة الدراسة بحسب استخدام تطبيقات الذكاء الاصطناعي في في التدريس. 
جدول ( ) : توزيع أفراد العينة من معلمات معاهد النور بحسب الاستخدام

\begin{tabular}{|c|c|c|c|}
\hline النسب المئوية & تكرارات & المستوى & المتغير \\
\hline$\% 00, \varepsilon$ & 10 & استخدم & \multirow[t]{3}{*}{ درجة استخدام تطبيقات الذكاء } \\
\hline$\% \varepsilon \varepsilon, 0$ & ir & لا استخدم & \\
\hline$\% \ldots$ & TV & المجموع & \\
\hline
\end{tabular}

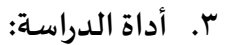

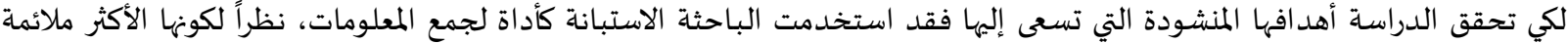

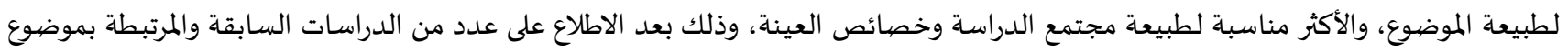

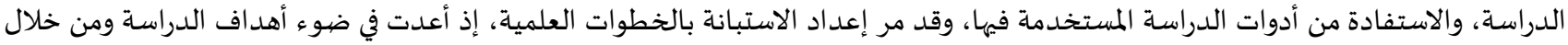
الإطار النظري والدراسات السابقة وآراء المحكمين.

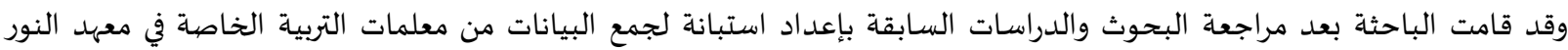

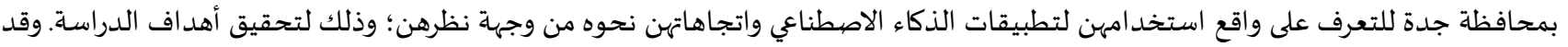
تكونت أداة الدراسة من جزءين: الجزء الأول: تضمن بيانات شخصية حول المعلمات عينة الدراسة، وتضم أسئلة حول: التخصص، وسنوات الخدمة، ودرجة استخدام تطبيقات الذكاء الاصطناعي في التدريس. الجزء الثاني: وتضمن (.ـ) فقرة موزعة على أربعة محاور كالتالي:

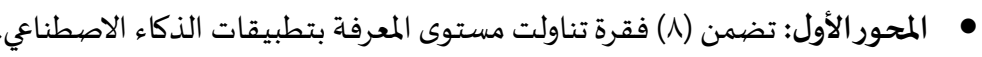

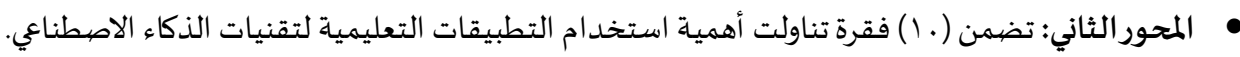
• • المحور الثالث: تضمن (10) فقرة تناولت معوقات استخدام التطبيقات التعليمية لتقنيات الذكاء الاصطناعي. • المحور الر ابع: تضمن (V) فقرات تناولت الاتجاه نحو استخدام التطبيقات التعليمية لتقنيات الذكاء الاصطناعي.

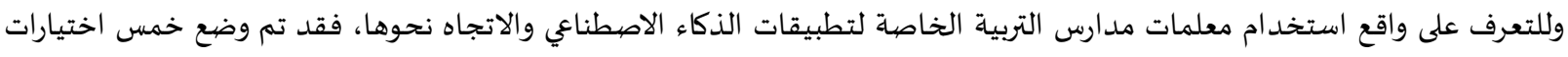

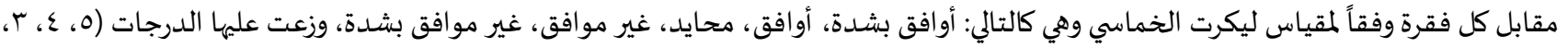

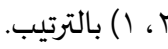

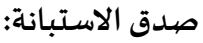

تتصف الأداة بأنها صادقة إذا ثبتت قدرتها على قياس ما وضعت لقياسه، وقد تم التحقق من صدق الاستبانة على النحو التالي:

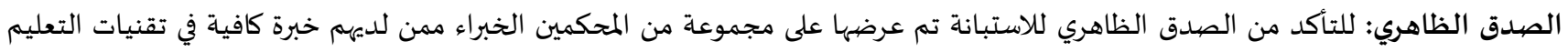

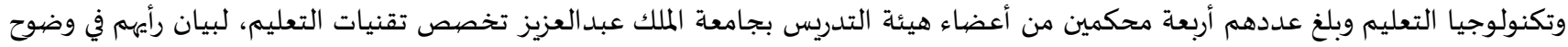

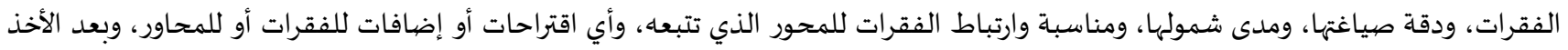

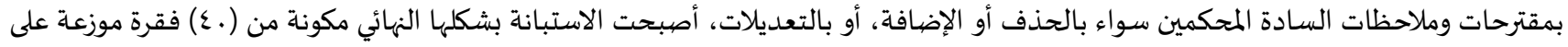

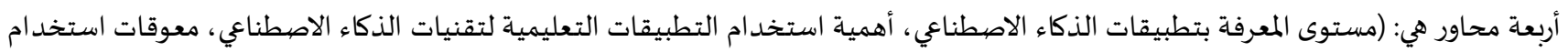

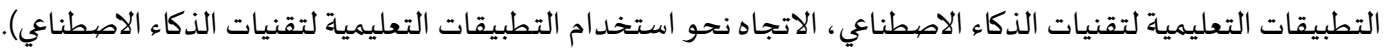

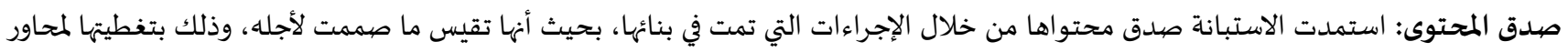
الاستبانة الأربعة.

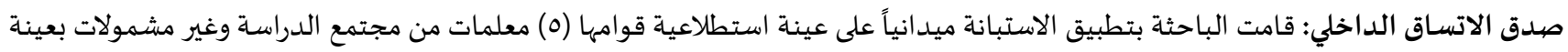
الدراسة من مدارس التربية الخاصة، وذلك للتحقق من صدق الاتساق الداخلي من خلال حساب معامل الارتباط لبيرسون (Pearson Correlation)

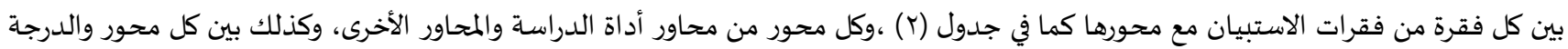

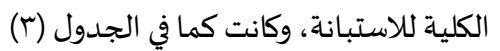


جدول (Y): معاملات الارتباط بين درجة كل فقرة من فقرات الاستبيان

\begin{tabular}{|c|c|c|c|c|c|}
\hline الدلالة الإحصيائية & معامل ارتباطها للبعد المنتمية له & رقم الفقرة & الدلالة الإحصيائية & معامل ارتباطها للبعد المنتمية له & رقم الفقرة \\
\hline 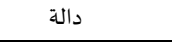 & ${ }^{* *} \cdot, \lambda 1$ & r & \multicolumn{3}{|c|}{ المحور الأول: مستوى المعرفة بالتطبيقات } \\
\hline 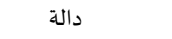 & $* * ., \wedge$ & $r$ & 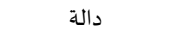 & ${ }^{* *} \cdot, \mathrm{V \wedge}$ & 1 \\
\hline دالة & $* * ., \vee \wedge$ & $\varepsilon$ & دالة & $* *, \wedge \vee$ & r \\
\hline 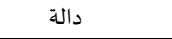 & ${ }^{* *} ., \wedge 1$ & $\circ$ & 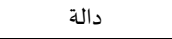 & ${ }^{* *} ., \mathrm{V} \vee$ & $r$ \\
\hline 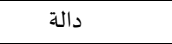 & $* * ., \wedge \vee$ & 7 & 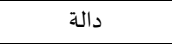 & $* * ., \Lambda 1$ & $\varepsilon$ \\
\hline 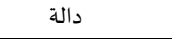 & ${ }^{* *} .$, , $\wedge$ & v & 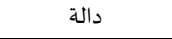 & $* * ., \wedge$. & 0 \\
\hline 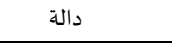 & **., $\vee$ १ & $\wedge$ & 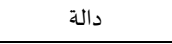 & **., V9 & 7 \\
\hline 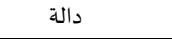 & **., Аr & 9 & 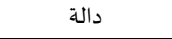 & $*^{* *}, \mathrm{\vee} \wedge$ & v \\
\hline 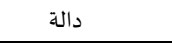 & ${ }^{* *} ., \mathrm{\vee} \wedge$ & 1. & 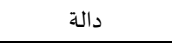 & **., TV & $\wedge$ \\
\hline 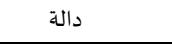 & **., ג个 & 11 & \multicolumn{3}{|c|}{ المحور الثاني: أهمية تطبيقات الذكاء } \\
\hline 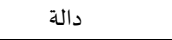 & $* * ., \vee\urcorner$ & ir & 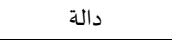 & $* * ., 1$ & 1 \\
\hline 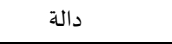 & **., V। & 15 & 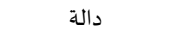 & $* * ., V Y$ & r \\
\hline 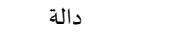 & $* * ., V r$ & $1 \varepsilon$ & 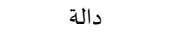 & $* * .,{ }_{1}$ & r \\
\hline 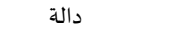 & $* * ., \wedge \varepsilon$ & 10 & 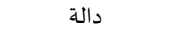 & $* * ., \wedge \varepsilon$ & $\varepsilon$ \\
\hline \multicolumn{3}{|c|}{ المحور الر ابع: الاتجاه نحو التطبيقات } & 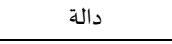 & ${ }^{* *}$, , $\mathrm{\wedge}$ & 0 \\
\hline 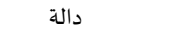 & $* * ., \mathrm{V}$ & 1 & 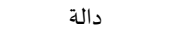 & $* * ., \vee 7$ & 7 \\
\hline 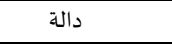 & **., ^乏 & $r$ & دالة & **., Vo $_{0}$ & v \\
\hline دالة & **., 79 & $r$ & دالة & ${ }^{* *} \cdot, \wedge \Lambda$ & $\wedge$ \\
\hline 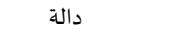 & $* * ., \wedge 0$ & $\varepsilon$ & 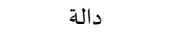 & $* * ., \vee 9$ & 9 \\
\hline 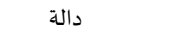 & **., ท9 & 0 & 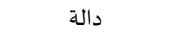 & ${ }^{* *} \cdot$, , ० & 1. \\
\hline 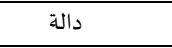 & $* * \cdot, \wedge 1$ & 7 & \multicolumn{3}{|c|}{ المحور الثالث: معوقات استخدام التطبيقات } \\
\hline 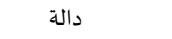 & $* * ., 17$ & v & 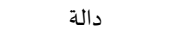 & **.,人ז & 1 \\
\hline
\end{tabular}

| * * دالة عند مستوى دلالة (1 ., (.)

يتبين من جدول (r) أن كل فقرة من فقرات الاستبيان أظهرت معامل ارتباط لها مع المحور المنتمية إليه، وأن قيم معاملات الارتباط بين درجة

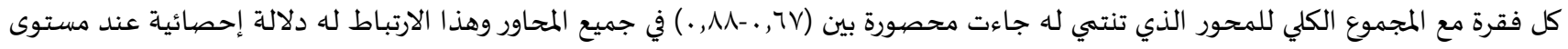

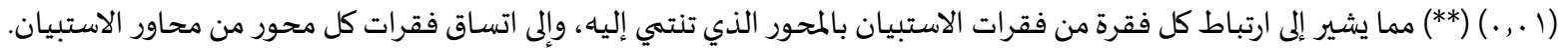

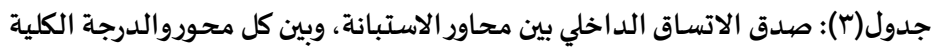

\begin{tabular}{|c|c|c|c|c|c|}
\hline الدرجة الكلية & الاتجـاه نحو & معوقات استخدام & أهمية تطبيقات & مستوى المعرفة & المحور \\
\hline **., ᄉ १1 & $* * ., \wedge \vee\urcorner$ & **.,人ґへ & **., VIr & 1 & مستوى المعرفة \\
\hline$* * ., 9 \leq \varepsilon$ & $* * ., \wedge 11$ & **.,VAr & 1 & **.,VIr & أهمية تطبيقات الذكاء \\
\hline **., qrv & $* *,, \vee 90$ & 1 & **., VAr & **.,人ץへ & معوقات استخدام \\
\hline **.,91ध & 1 & **., $\vee 190$ & $* * ., \wedge \backslash 1$ & **., АVฯ & الاتجاه نحو التطبيقات \\
\hline 1 & **., १। $\varepsilon$ & **., qrv & $* * ., 9 \leq \varepsilon$ & $* * ., \wedge 91$ & الدرجة الكلية \\
\hline
\end{tabular}

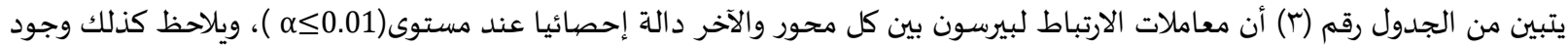

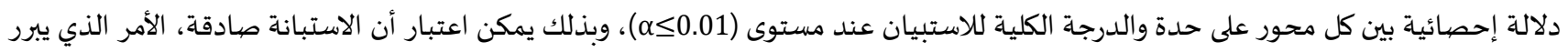
استخدامها لأغراض هذه الدراسة، ويزيد الثقانة الباحثة بها.

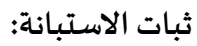
تم حساب ثبات أداة الدراسة (الاستبانة) بطريقتين:

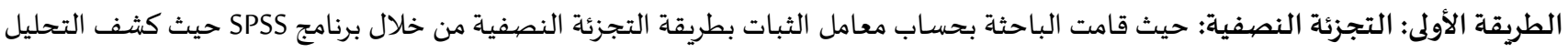

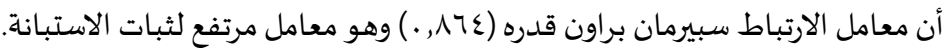

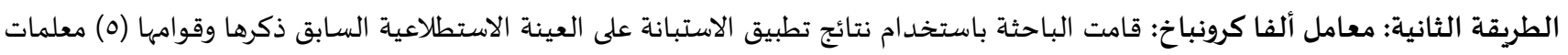

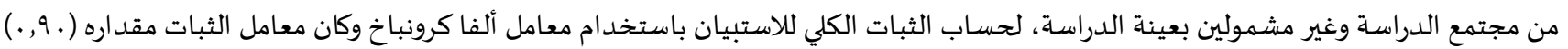
وهو معامل ثبات مرتفع واعتبرته الباحثة كافياً لأغراض هذه الدراسة. 
جدول (ع): حساب الثبات باستخدام معامل ألفا لكرونباخ والتجزئة النصفية لسبيرمان براون

\begin{tabular}{|c|c|c|c|}
\hline معامل التجزئة النصيفية & معامل ألفا لكرونباخ & 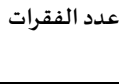 & المحتور \\
\hline . &., $0 \leqslant 9$ & $\wedge$ & مستوى المعرفة والمهارة المرتبطة باستخدام الذكاء الاصطناعي \\
\hline ., VVr & , vor & 1. & أهمية استخدام التطبيقات التعليمية لتقنيات الذكاء الاصطناعي \\
\hline . & . & 10 & معوقات استخدام التطبيقات التعليمية لتقنيات الذكاء الاصطناعي \\
\hline , ArI & , Nor & $\mathrm{r}$ & الاتجاه نحو استخدام التطبيقات التعليمية لتقنيات الذكاء الاصطناعي \\
\hline .,, $0 \varepsilon$ &., 9 . & . ع فقرة & الثبات العام للاستبيان \\
\hline
\end{tabular}

إجراءات تطبيق أداة الدراسة:

تم توزيع الاستبانة على عينة الدراسة للإجابة عليها في شهر ربيع أول من العام اعـا هـ، ونظراً لرغبة الباحثة في الخروج بنتائج تخدم موضوع

الدراسة قدر المستطاع، فقد قامت الباحثة بتوزيع الاستبانة إلكترونياً على جميع معلمات التربية الخاصة في معهد النور بمحافظة جدة.

قامت الباحثة بجمع استجابات المعلمات الإلكترونية ثم قامت الباحثة بتفريغها لإجراء التحليلات اللازمة.

نتائج البحث ومناقشتها:

قامت الباحثة في هذا الفصل بالإجابة على السؤال الرئيس للبحث والمتمثل في " ما واقع استخدام تطبيقات الذكاء الاصطناعي في مدارس التربية

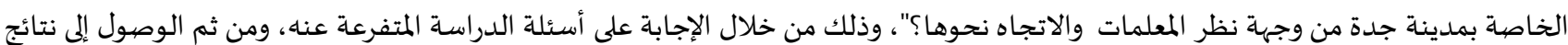

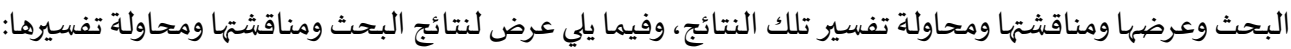

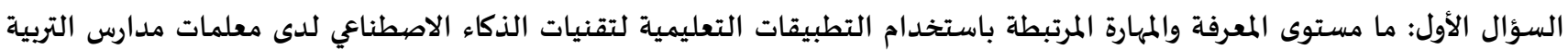

الخاصة من وجهة نظرهن؟

للإجابة عن سؤال البحث الأول تم حساب المتوسطات الحسابية والانحرافات المعيارية لاستجابات أفراد العينة على استبانة واقع استخدام معلمات مدارس التربية الخاصية لتطبيقات الذكاء الاصطناعي والاتجاه نحوها في محور (مستوى المعرفة والمهارة المرتبطة باستخدام التطبيقات التعليمياة لتقنيات الذكاء الاصطناعي)، والجدول رقم ( 0) يوضح هذه النتائج.

جدول(0): المتوسطات الحسابية والانحر افات المعيارية لفقرات و اقع استخدام معلمات مدارس التربية الخاصة لتطبيقات الذكاء الاصطناعي والاتجاه نحوها في محور (مستوى المعرفة والمهارة المرتبطة باستخدام التطبيقات التعليمية لتقنيات الذكاء الاصطناعي) من وجهه نظر معلمات مدارسات الماتس التربية الخاصية

\begin{tabular}{|c|c|c|c|c|c|}
\hline 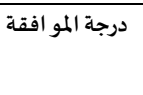 & المعياري & الحسابي المتوسط & 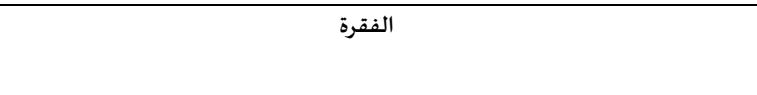 & 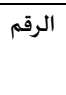 & الرتبة \\
\hline محايد & $1, \cdot r \Lambda$ & r, rer & أعرف المفاهيم والمصطلحات المتعلقة بتقنيات الذكاء الاصطناعي. & 1 & r \\
\hline 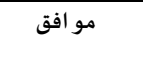 & $1, . \varepsilon r$ & $r, r v$ & أخثق في قدر اتي على التعرف على تطبيقات الذكاء الاصطناعي وتوظيفها لطالبات التربية & r & r \\
\hline 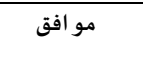 & $\cdot$, , A & $r, \varepsilon \lambda$ & 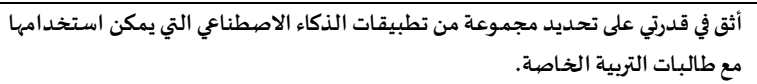 & r & 1 \\
\hline محايد & $1,1 M 1$ & $r, 11$ & أعرف كيفية استخدام العديد من تطبيقات الذكاء الاصطناعي لمساعدة طالبات التربية & $\varepsilon$ & $\varepsilon$ \\
\hline 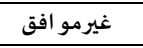 & $1,1 \% 1$ & $r, 07$ & اتبع خطة منهجية للتاكد من فعالية استخدام تطبيقات الذكاء الاصطناعي. & $\circ$ & v \\
\hline محايد &., $\mathrm{V} \ \varepsilon$ & $r, Y \varepsilon$ & لدي القدرة على إيجاد مصيادر التطوير المتعلقة بتقنيات الذكاء الاصطناعي. & 7 & $\circ$ \\
\hline 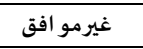 &., $9 \times 1$ & $r, 09$ & أستطيع حل المشكلات التقنية التي تواجني عند استخدام تطبيقات الذكاء الاصطناعي. & $\mathrm{v}$ & 7 \\
\hline 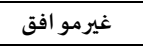 & $1,1 \leqslant 1$ & $r, \cdot V$ & تلقيت تدريبأ كافيًا على استخدام تقنيات الذكاء الاصطناعي أثناء العمل & $\wedge$ & $\wedge$ \\
\hline 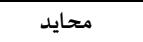 &., $\mathrm{VOA}$ & $r, q 1$ & المتوسط الحسابي للمجال ككل & & \\
\hline
\end{tabular}

أشارت نتائج الجدول رقم ( 0) أن المتوسط الحسابي العام لاستجابات عينة البحث يساوي (1, (1)، وهو مؤشر على أن هناك موافقة بدرجة (محايد) على فقرات واقع استخدام معلمات مدارس التربية الخاصة لتطبيقات الذكاء الاصطنائ والاتجاه نحوها في محور (مستوى المعرفة والمهارة

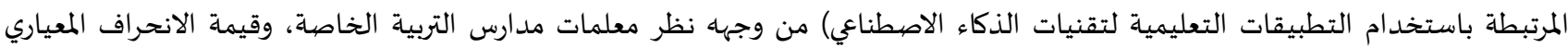
للمتوسط الحسابي العام للمجال يساوي (مهV, •)، وهي قيمة ومؤشر على التجانس الكبير بين استجابات عينة البحث حول واقع استخدام معلمات 
مدارس التربية الخاصة لتطبيقات الذكاء الاصطناعي والاتجاه نحوها في محور (مستوى المعرفة والمهارة المرتبطة باستخدام التطبيقات التعليمية لتقنيات الذكاء الاصطناعي) من وجهاه نظر معلمات مدارس التربية الخاصية.

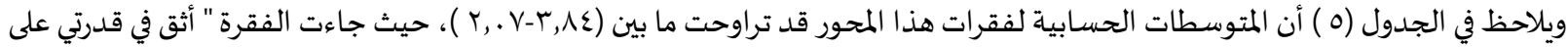

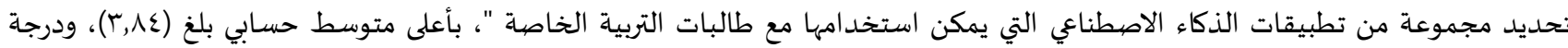
موافقة (موافق)، وجاءت الفقرة " أثق في قدراتي على التعرف على تطبيقات الذكاء الاصطناعي وتوظيفها لطالبات التربية الخاصة" بمتوسط حسابي بلغ

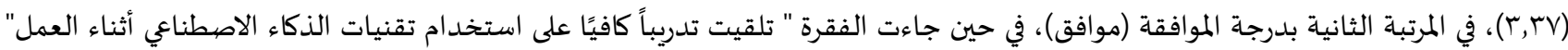
بأدنى متوسط حسابي بلغ (V, . . )، ودرجة الموافقة (غير موافق)، كما تشير النتائج إلى أن بقية الفقرات لهذا المحور قد تراوحت بين درجة (موافق- غير

السؤال الثاني: ما أهمية استخدام معلمات مدارس التربية الخاصية للتطبيقات التعليمية لتقنيات الذكاء الاصطناعي من وجهة نظرهن؟

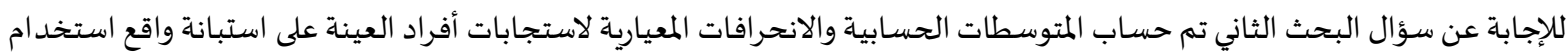
معلمات مد ارس التربية الخاصة لتطبيقات الذكاء الاصطناعي والاتجاه نحوها في محور (أهمية استخدام معلمات مدارس التربية الخاصية للتطبيقات التعليمية لتقنيات الذكاء الاصطناعي)، والجدول رقم ( آ) يوضح هذه النتائج.

جدول ( 7): المتوسطات الحسابية والانحر افات المعيارية لفقرات و اقع استخدام معلمات مدارس التربية الخاصة لتطبيقات الذكاء الاصطناعي والاتجاه نحوها في محور (أهمية استخدام معلمات مدارس التربية الخاصة للتطبيقات التعليمية لتقنيات الذكاء الاصطناعي) من وجهاته نظرهن

\begin{tabular}{|c|c|c|c|c|c|}
\hline 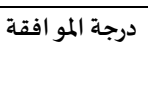 & 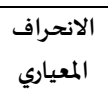 & المتوسط المسبي & 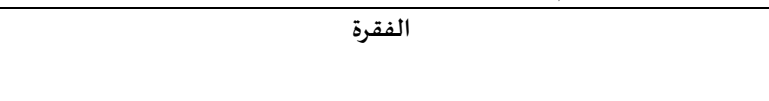 & 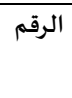 & 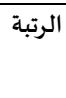 \\
\hline 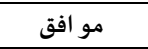 & $\cdot$, , $9 \mathrm{r}$ & $r, \wedge 9$ & تسهل تطبيقات الذكاء الاصطناعي إنجاز الأعمال بأقل وقت وجهد ممكن. & 1 & $\wedge$ \\
\hline مو افق & ع & $r, \wedge q$ & تمعاهم تقنيات الذكاء الاصطناعي في تغيير دور الطالب من متلق للمعرفة إلى باحث عن & r & $\wedge$ \\
\hline 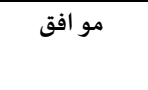 & $\cdot$, T. & $\varepsilon, 10$ & والمزلمات توظيف تقنيات الذكاء الاصطناعي في التعلم من التواصل بين الطالبات & $r$ & 7 \\
\hline 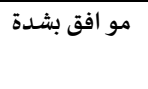 & .,01. & $\varepsilon, 0$ Y & تقلية التعلم (الخجل مثلئلا). & $\varepsilon$ & r \\
\hline 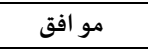 & $\cdot, \mathrm{V} r \mathrm{~T}$ & $\varepsilon, 19$ & تساهم تقنيات الذكاء الاصطناعي في تنمية المهارات البحثية لدى الطالبات. & 0 & 0 \\
\hline 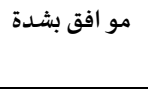 &., $0 \leqslant r$ & $\varepsilon, r$. & تساهم التطبيقات التعليمية لتقنيات الذكاء الاصطناعي في تنمية مهارات التفكير لدى & 7 & $\varepsilon$ \\
\hline 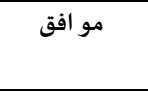 & , V01 & $\{, 11$ & تين الطالبات. & $\checkmark$ & v \\
\hline 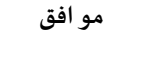 & ., VA & $\varepsilon, 19$ & 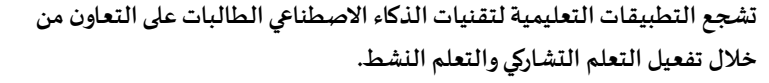 & $\wedge$ & 0 \\
\hline 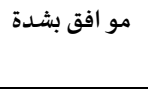 &., $0 \leqslant Y$ & $\varepsilon, \gamma$. & تضغيفي تقنيات الذكاء الاصطناعي نوع من الحيوية والجاذبية على عرض المادة & 9 & 1 \\
\hline 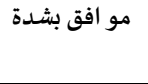 & 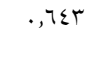 & $\varepsilon, \varepsilon \wedge$ & تحفز التطبيقات التعليمية لتقنيات الذكاء الاصطناعي الطالبات على زيادة مستوى & 1. & r \\
\hline مو افق بشـدة & או & $\varepsilon, \varepsilon T$ & المتوسط الحسابي للمجال ككل & & \\
\hline
\end{tabular}

أشـارت نتائج الجدول رقم ( 7) أن المتوسط الحسابي العام لاستجابات عينة البحث يساوي (ع ؟ ع)، وهو مؤشر على أن هناك موافقة بدرجة (موافق بشدة) على فقرات واقع استخدام معلمات مدارس التربية الخاصة لتطبيقات الذكاء الاصطناعي والاتجاه نحوها في محور (أهمية استخدام

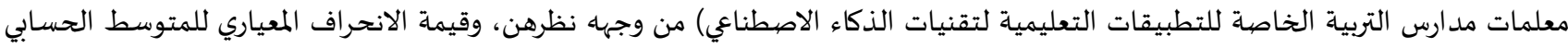

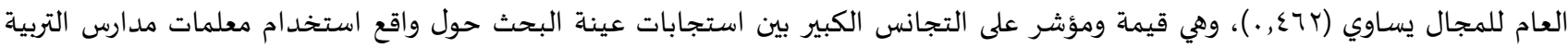
الخاصاة لتطبيقات الذكاء الاصطناعي والاتجاه نحوها في محور (أهمية استخدام معلمات مدارس التربية الخاصة للتطبيقات التعليمية لتقنيات الذكاء

الاصطناعي) من وجهاه نظرهن.

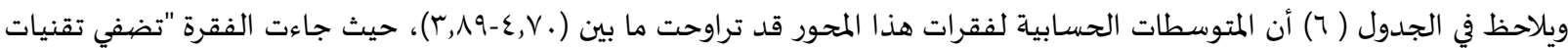
الذكاء الاصطناعي نوع من الحيوية والجاذبية على عرض المادة التعليمية "، بأعلى متوسط حسابي بلغ (.V,V))، ودرجة موافقة (موافق بشدة)، وجاءت

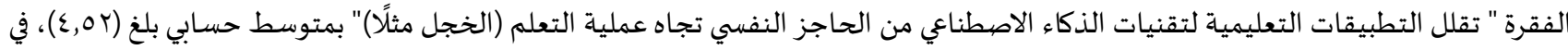


المرتبة الثانية بدرجة الموافقة (موافق بشدة)، في حين جاءت الفقرة "تتسهل تطبيقات الذكاء الاصطناعي إنجاز الأعمال بأقل وقت وجهد ممكن " بأدنى (مأى

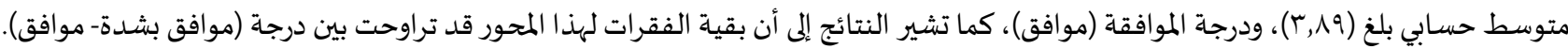
السؤال الثالث: ما معوقات استخدام التطبيقات التعليمية لتقنيات الذكاء الاصطناعي لدى معلمات مدارس التربية الخاصة من وجهة نظرهن؟ للإجابة عن سؤال البحث الثالث تم حساب المتوسطات الحسابية والانحرافات المعيارية لاستجابات أفراد العينة على استبانة واقع استخدام معلمات مدارس التربية الخاصة لتطبيقات الذكاء الاصطناعي والاتجاه نحوها في محور (معوقات استخدام التطبيقات التعليمية لتقنيات الذكاء الاصطناعي)، والجدول رقم (V) يوضح هذه النتائج.

جدول (V): المتوسطات الحسابية والانحر افات المعيارية لفقرات و اقع استخدام معلمات مدارس التربية الخاصة لتطبيقات الذكاء الاصطناعي

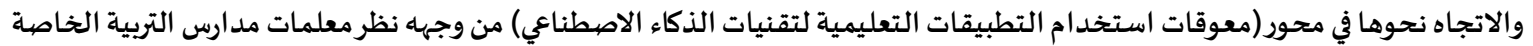

\begin{tabular}{|c|c|c|c|c|c|}
\hline 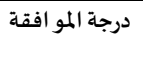 & 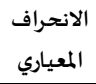 & الحستوسط & 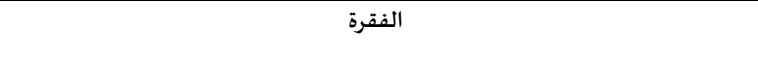 & 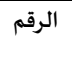 & 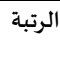 \\
\hline محايد & 1,497 & r, rT & مقاومة استخدام تقنيات الذكاء الاصطناعي من قبل المعلمات في الممدرسة. & 1 & $1 \varepsilon$ \\
\hline 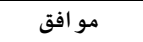 & $\cdot, \wedge 7 \varepsilon$ & $\varepsilon, 10$ & قلة الوعي لدى بعض أصحاب القرارفي المدارس بأهمية تقنيات الذكاء الاصطناعي. & r & 1 \\
\hline 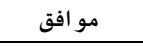 & $1, .0$ & $r, \mathrm{rA}$ & التطبيقات العربية التي تخدم التعليم غيركافية في تقنيات الذكاء الاصطناعي. & $r$ & 1 \\
\hline 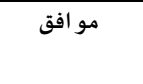 & $1,|r|$ & $r, \wedge 9$ & المستهدفة من ذوب التبيقات التعليمية لتقنيات الذكاء الاصطناعي المتوفرة مع خصائص الفئة & $\varepsilon$ & 1 \\
\hline 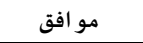 & $\cdot, 109$ & $r, v \varepsilon$ & عدم وجود الخبرة الكافية للتعامل مع تقنيات الذكاء الاصطناعي من قبل المعلمات. & $\circ$ & 9 \\
\hline 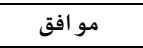 & $1, \ldots 1$ & $r, \Lambda 1$ & الحاجة لتدربب المعلمات على استخدام تقنيات الذكاء الاصطناعي. & 7 & $\mathrm{r}$ \\
\hline 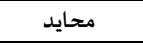 &., $9 \cdot 4$ & $r, r]$ & صعوبة التعامل مع تقنيات الذكاء الاصطناعي في العملية التعليمية. & $\mathrm{v}$ & ir \\
\hline محايد & $1,1 \leq \varepsilon$ & $r, r r$ & تقنيات الذكاء الاصطناعي غيرمناسبة لطرق التدريس الحالية المستخدمة بالمدارس. & $\Lambda$ & 11 \\
\hline محايد & 1,rro & $r, r$. & الطالبات. استخدام تقنيات الذكاء الاصطناعي داخل القاعة الدراسية سيكون عاملا مشتتا لأذهان & 9 & ir \\
\hline 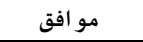 & $1, \varepsilon 1 Y$ & $r, q 4$ & ضبعف البنية التكنولوجية الأساسية في بعض المدارس. & 1. & . \\
\hline 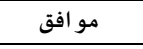 &., 901 & $\varepsilon, \cdot v$ & الدعم الفني المقدم لا يتناسب مع أهمية استخدام المعلمات لتقنيات الذكاء الاصطناعي. & 11 & r \\
\hline 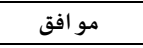 & 1,117 & $r, 97$ & لا يوجد حو افزمادية أو معنوية لمن يستخدم تقنيات الذكاء الاصطناعي في التعليم. & ir & $\varepsilon$ \\
\hline 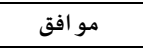 & $1, r \varepsilon$ & $r, \varepsilon \varepsilon$ & لا امتلك الوقت الكافي لاستخدام تقنيات الذكاء الاصطناعي في التعليم. & ir & 1. \\
\hline 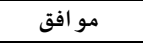 &., $\mathrm{AVV}$ & $\varepsilon, .$. & ارتفاع أسعار تقنيات الذكاء الاصطناعي يحول دون قدرة المدارس على شرائها. & $1 \varepsilon$ & $r$ \\
\hline محايد & $1, r 10$ & $r, . \varepsilon$ & أشعربالقلق من استخدام تطبيقات تعتمد على تقنيات الذكاء الاصطناعي في التدربس. & 10 & 10 \\
\hline مو افق & . גir. & $r, 77$ & المتوسط الحسابي للمجال ككل & & \\
\hline
\end{tabular}

أشارت نتائج الجدول رقم (V) أن المتوسط الحسابي العام لاستجابات عينة البحث يساوي (r,77)، وهو مؤشر على أن هناك موافقة بدرجة (موافق) على فقرات واقع استخدام معلمات مدارس التربية الخاصة لتطبيقات الذكاء الاصطناعي والاتجاه نحوها في محور (معوقات استخدام

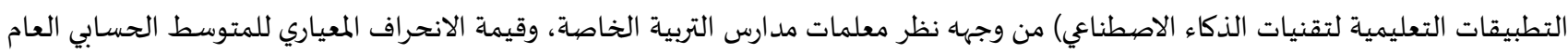
للمجال يساوي (. rی, •)، وهي قيمة ومؤشر على التجانس الكبير بين استجابات عينة البحث حول واقع استخدام معلمات مدارس التربية الخاصية

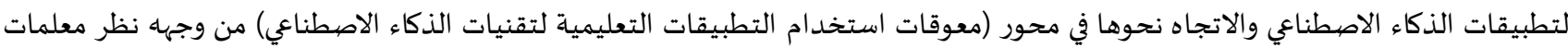
مدارس التربية الخاصة.

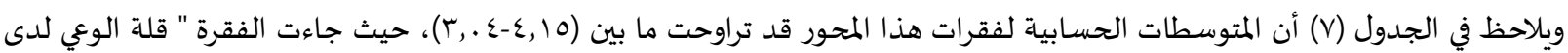
بعض أصحاب القرار في المدارس بأهمية تقنيات الذكاء الاصطناعي "، بأعلى متوسط حسابي بلغ (10,ع)، ودرجة موافقة (موافق)، وجاءت الفقرة "

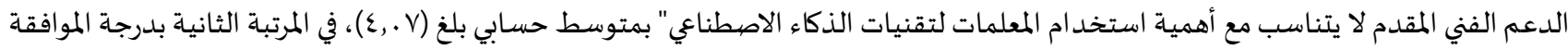
(موافق)، في حين جاءت الفقرة " أشعر بالقلق من استخدام تطبيقات تعتمد على تقنيات الذكاء الاصطناعي في التدريس" بأدنى متوسط حسابي بلغ (ع . ب)، ودرجة الموافقة (محايد)، كما تشير النتائج إلى أن بقية الفقرات لهذا المحور قد تراوحت بين درجة (موافق -محايد).

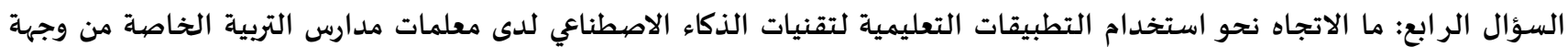

نظرهن؟

للإجابة عن سؤال البحث الرابع تم حساب المتوسطات الحسابية والانحرافات المعيارية لاستجابات أفراد العينة على استبانة واقع استخدام معلمات مد ارس التربية الخاصة لتطبيقات الذكاء الاصطناعي والاتجاه نحوها في محور (الاتجاه نحو استخدام التطبيقات التعليمية لتقنيات الذكاء

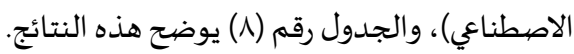


جدول (^): المتوسطات الحسابية والانحر افات المعيارية لفقرات و اقع استخدام معلمات مدارس التربية الخاصة لتطبيقات الذكاء الاصطناعي والاتجاه نحوها في محور (الاتجاه نحو استخدام التطبيقات التعليمية لتقنيات الذكاء الاصطناعي) من وجهه نظر معلمات مدارس الترات التربية الخاصية

\begin{tabular}{|c|c|c|c|c|c|}
\hline 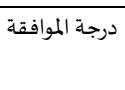 & 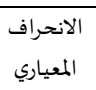 & الحسابي & 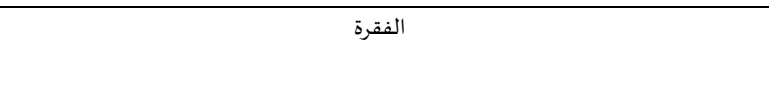 & 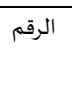 & 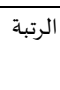 \\
\hline 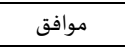 & $1,1 \mathrm{~V} \Lambda$ & $r, \Lambda 1$ & أعتقد أن تقنيات الذكاء الاصطناعي ستساعدني على متابعة أداء الطالبات وانجازهم. & 1 & 1 \\
\hline 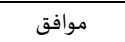 & $1, .17$ & $r, \mathrm{r} \Lambda$ & أعتقد أن تقنيات الذكاء الاصطناعي ستكون أداة تواصل واتصال بيني وبين طالباتي. & r & r \\
\hline 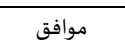 &., 974 & $r, \Lambda)$ & أرى أن استخدام تقنيات الذكاء الاصطناعي سيجعل من عملية التدريس أكثر فاعلية وتفاعلية. & $r$ & 1 \\
\hline 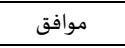 & $\cdot, 109$ & $r, \mathrm{r} \varepsilon$ & أحب التدريس باستخدام تطبيقات تعتمد على تقنيات الذكاء الاصطنائ. & $\varepsilon$ & $r$ \\
\hline موافق & $1,1 \vee \lambda$ & $r, \Lambda 1$ & أرى أن استخدام تقنيات الذكاء الاصطناعي في التعليم سيساعد في زيادة دافعية الطالبات. & $\circ$ & 1 \\
\hline محايد & $1, r / 9$ & $r, \mathrm{rA}$ & أرى أن المقررات الحالية التي أدرسها معدة لتوظيف تقنيات الذكاء الاصطناعي في التدريس. & 7 & 0 \\
\hline موافق & $\cdot, \curlyvee \wedge \varepsilon$ & $r, \mathrm{TV}$ & أنصح زميلاتي المعلمات باستخدام تقنيات الذكاء الاصطناعي في التعليم لأهها أكثر من مجرد & $\mathrm{v}$ & $\varepsilon$ \\
\hline 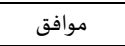 & , Nor & $r, 7 r$ & المتوسط الحسابي للمجال ككل & & \\
\hline
\end{tabular}

أشارت نتائج الجدول رقم (^) أن المتوسط الحسابي العام لاستجابات عينة البحث يساوي (rآ, )، وهو مؤشر على أن هناك موافقة بدرجة

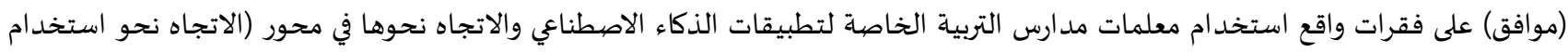

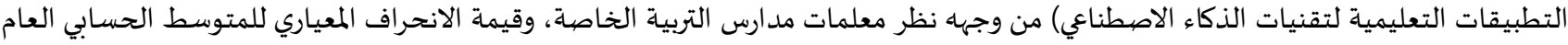

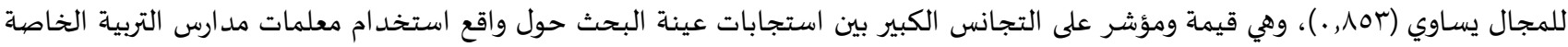
لتطبيقات الذكاء الاصطناعي والاتجاه نحوها في محور (الاتجاه نحو استخدام التطبيقات التعليمية لتقنيات الذكاء الاصطناعي) من وجهاه نظر معلمات مدارس التربية الخاصة.

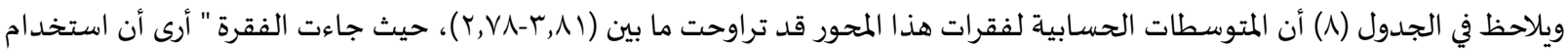

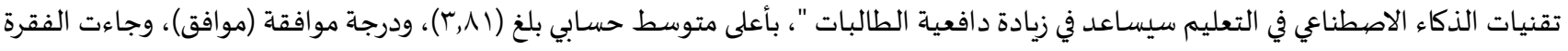

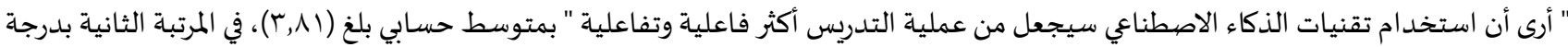

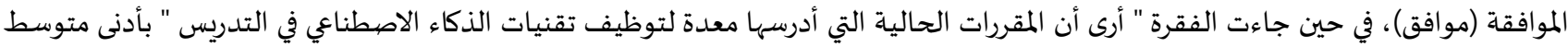

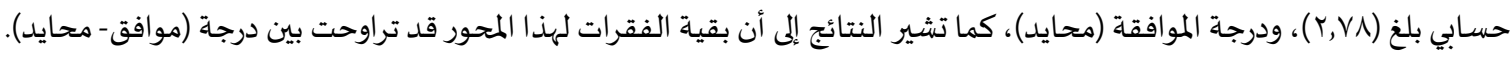

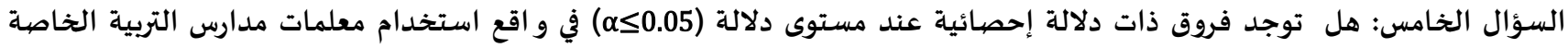

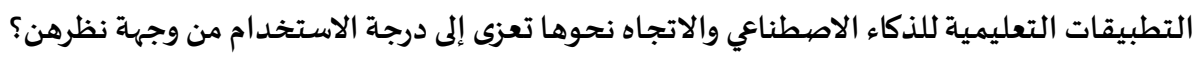

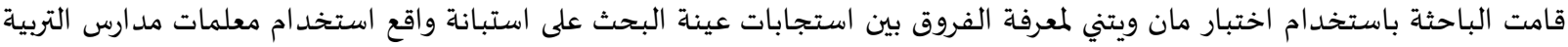

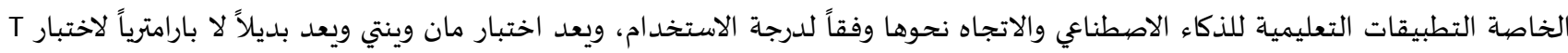
لعينيتين مستقلتين، ويوضح جدول (9) النتائج.

جدول (9): اختبار مان وينتي لمعرفة الفروق بين استجابات عينة البحث على استبانة و اقع استخدام معلمات مدارس التربية الخاصية التطبيقات

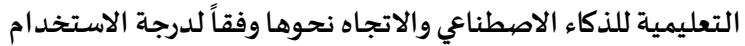

\begin{tabular}{|c|c|c|c|c|c|c|c|c|}
\hline الدلالة & قيمة Z & قيمياة W & قيمة u & مجموع الرتب & متوسط الرتب & العدد & المجموعة & المحور \\
\hline \multirow{2}{*}{ دالة عند مستوى (0. . . .) } & \multirow[t]{2}{*}{$r, r \wedge q$} & \multirow[t]{2}{*}{$1,1, \ldots$} & \multirow[t]{2}{*}{$r r, \ldots$} & TVV & $\backslash \Lambda, \varepsilon V$ & 10 & استخدام & \multirow[b]{2}{*}{ المححور الأول } \\
\hline & & & & 1.1 & $\Lambda, \varepsilon r$ & ir & الاستخدام & \\
\hline \multirow{2}{*}{ 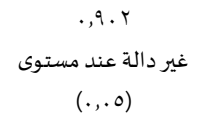 } & \multirow[t]{2}{*}{. ITE } & \multirow[t]{2}{*}{$r \cdot V, O \ldots$} & \multirow[t]{2}{*}{$\wedge \vee, O \ldots$} & $r \cdot v, O$. & $|\varepsilon, Y|$ & 10 & استخدام & \multirow[b]{2}{*}{ المحور الثاني } \\
\hline & & & & IV.,o. & Ir,人r & ir & الاستخدام & \\
\hline \multirow{2}{*}{ دالة عند مستوى (0. . . .) } & \multirow[t]{2}{*}{ r,IVA } & \multirow[t]{2}{*}{$1 \leq 0, \ldots$} & \multirow[t]{2}{*}{$r_{0}, \ldots$} & $1 \leqslant 0, .$. & $9,7 \mathrm{~V}$ & 10 & استخدام & \multirow[b]{2}{*}{ المحور الثالث } \\
\hline & & & & rrr,... & $19, \varepsilon T$ & ir & الاستخدام & \\
\hline \multirow{2}{*}{ دالة عند مستوى (ه ., . .) } & \multirow[t]{2}{*}{$r, q \vee \varepsilon$} & \multirow[t]{2}{*}{$1 \cdot v, 0 \ldots$} & \multirow[t]{2}{*}{$r q, 0 \ldots$} & $r V \cdot, O$. & $1 \Lambda, . r$ & 10 & استخدام & \multirow[b]{2}{*}{ المحور الرابع } \\
\hline & & & & $1 . V, 0$. & 1,97 & ir & الاستخدام & \\
\hline
\end{tabular}


المحور الأول: مستوى المعرفة والمهارة المرتبطة باستخدام التطبيقات التعليمية لتقنيات الذكاء الاصطناعي:

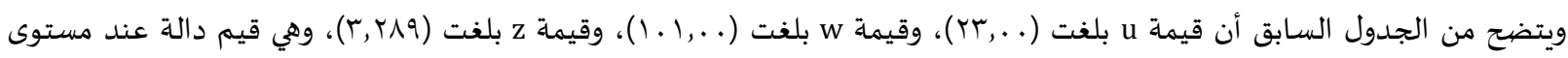

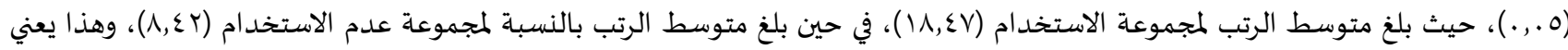

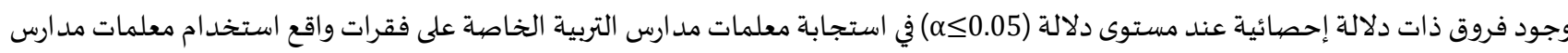
التربية الخاصة لتطبيقات الذكاء الاصطناعي والاتجاه نحوها في محور (مستوى المعرفة والمهارة المرتبطة باستخدام التطبية داتهات التعليمية لتقنيات الذكاء الاصطناعي) لصالح مجموعة استخدام تقنيات الذكاء الاصطناعي. وهذا يتشابه مع دراسة بنج مو (Ping Mu, 2019) إذ وجدت أن المهارة في الاستخد ام لا تزال محدودة وفي بداياتها ، ويمكن أن نفسر ذلك لكونها

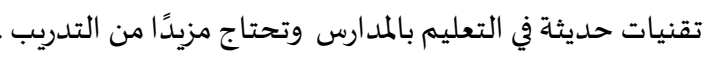
المحور الثاني: أهمية استخدام معلمات مدارس التربية الخاصية للتطبيقات التعليمية لتقنيات الذمات الذكاء الاصطنائي:

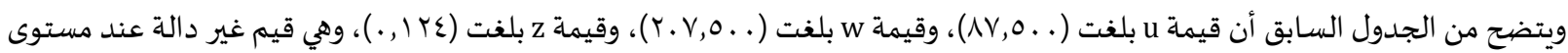

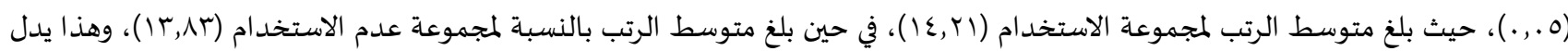

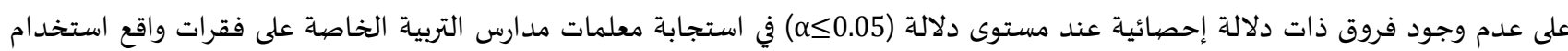

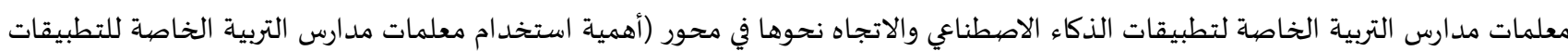
التعليمية لتقنيات الذكاء الاصطناعي). وهذا يوافق دراسة مونيكا وآخرين (Monica, et. al, 2018) ودراسة بوحصوان وآخرين (PO-HSUAN, et. al, 2018) للدور الفعال للتقنيات في تحسين أداء المتعلمين لإمكاناتها المتميزة.

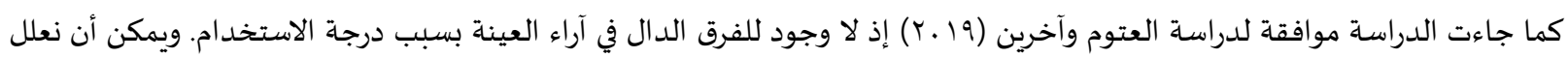
ذلك بأن التطبيقات التعليمية للذكاء الاصطناعي متوفرة في المعهد ، فأصبح استخداههن لها أمر واقعي؛ كون هذه الأجهزة تقدم خدمات للهودئ للمعلم

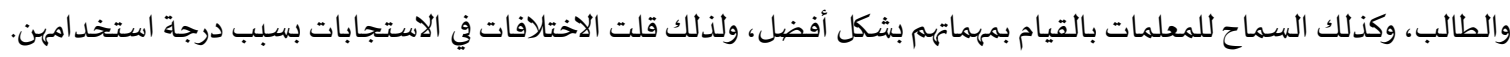

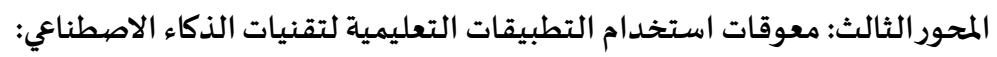

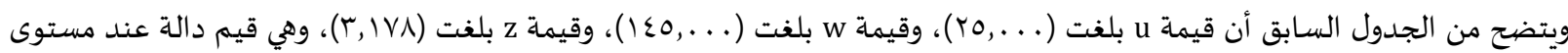

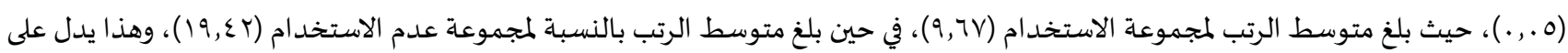

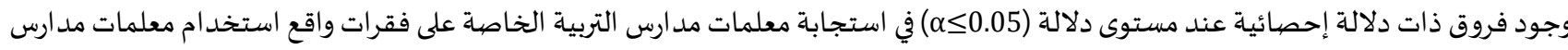

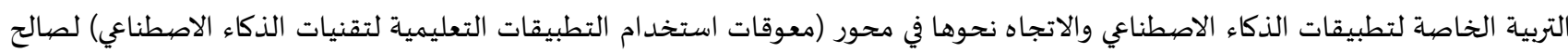
مجموعة عدم استخد ام تقنيات الذكاء الاصطناعي. وقد أظهرت دراسـات مثل دراسة مايكوي (Makoye, 2018) و دراسـة سعيد (Said, 2018) عن وجود عوائق كبيرة للاستخدام منها التكلفة

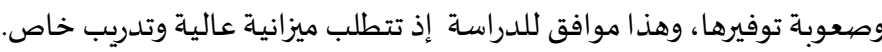

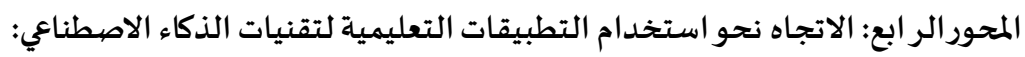

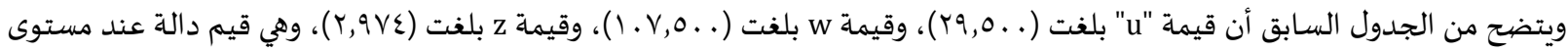

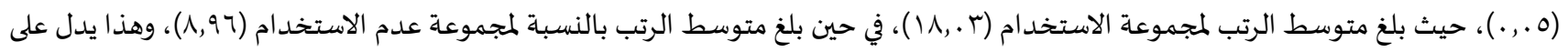

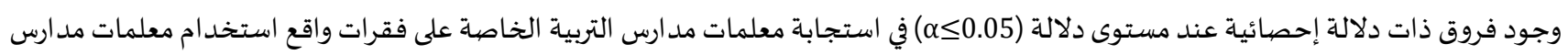
التربية الخاصاة لتطبيقات الذكاء الاصطناعي والاتجاه نحوها في محور (الاتجاه نحو استخدام التطبيقات التعليمية لتقنيات الذكاء الاصطيناعي) لصالح مجموعة استخدام تقنيات الذكاء الاصطناعي.

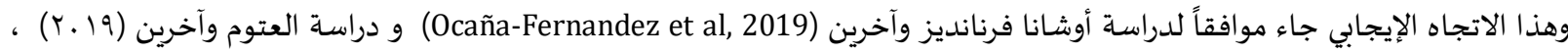

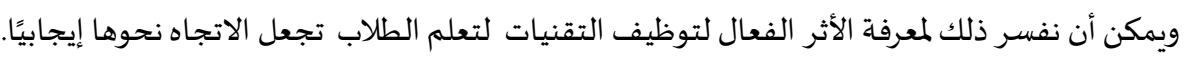

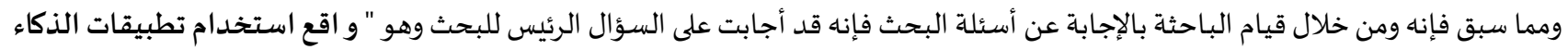

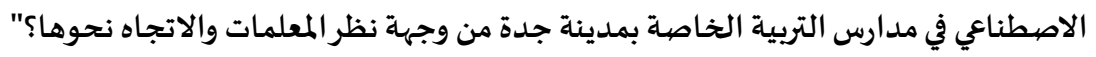
التوصيات والمقترحات: 1. الاهتمام بعمل أدلة لتوضيح آلية التدريس بتطبيق تقنيات قائمة على الذكاء الاصطناعي. r. تزويد أهل الاختصاص بوزارة التعليم بنتائج البحوث عن معوقات تطبيقات الذكاء الاصطناعي لتطوير مؤسساتها.

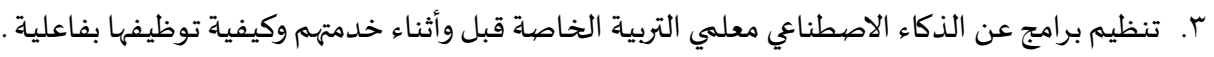


ع. تضمين تطبيقات الذكاء الاصطنايي بكافة أشكالها في المناهج الدراسية الخاصة بذوي الاحتياجات الخاصة بالمراحل التعليمية المختلفة وبخاصة في المراحل الدنيا. 0. إجراء الدراسات لتطبيقات الذكاء الاصطناعي حول فئات أخرى للتربية الخاصةة .

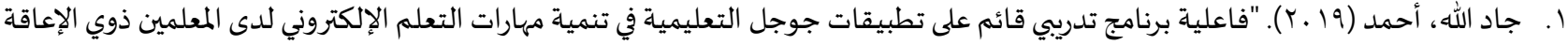

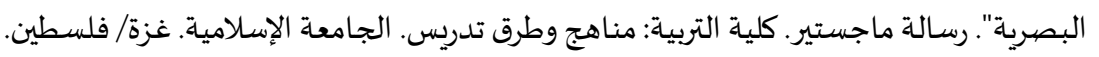

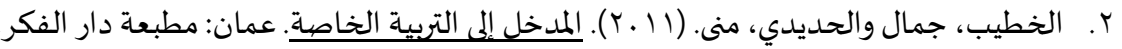

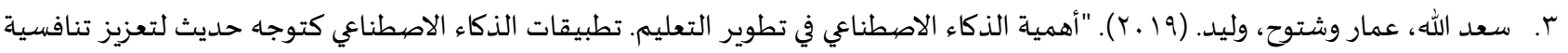
منظمات الأعمال"، برلين: المركز الديمقراطي العربي.

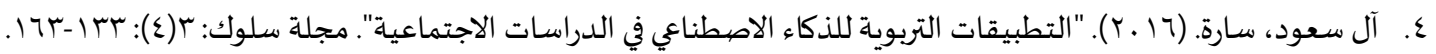
ه. السيد، هويدا. (عا ـ †). "فاعلية برنامج تدريبي إلكتروني في إكساب بعض مهارات استخدام مواقع التواصل الاجتماعي والثقة بالنفس والاتجاه

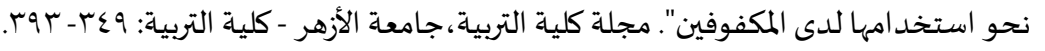

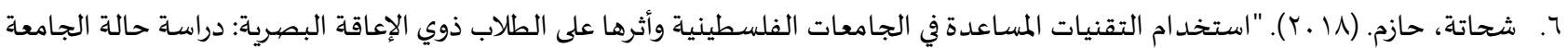

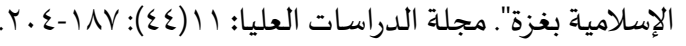
V. أبو شمالة، رشا. (rا . r). "فاعلية برنامج قائم على الذكاء الاصطناعي لتنمية التفكير الاستدلالي والتحصيل الدراسي في مبحث تكنولوجيا المعلومات لدى طالبات الحادي عشر بغزة". رسالة ماجستير بكلية التربية جامعة الأزهر. غزة.

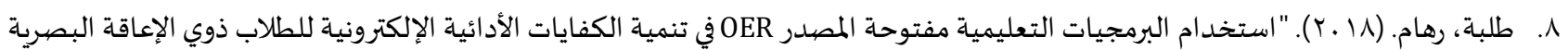
في مدرسة النور بمحافظة أسيوط". مجلة العلوم التربوية والنفسية، المجلة العربية للعلوم ونشر الأبحاث: ؟ (. 1): ا-10ب.

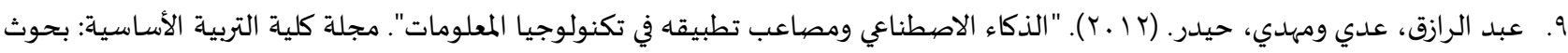

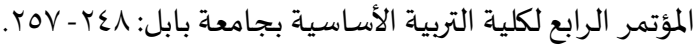

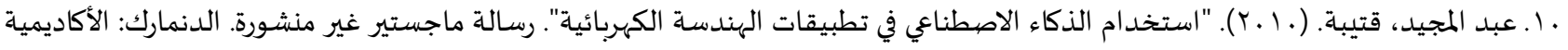
العربية. 11 ا. العتوم، نعيم وحتاملة، حابس والصمادي، علي. (19 .ب). "دور التقنيات المساندة الخاصة بالمكفوفين في تمكينهم الاجتماعي من وجهة نظرهم".

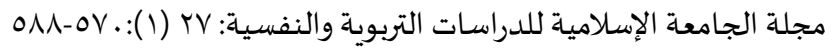

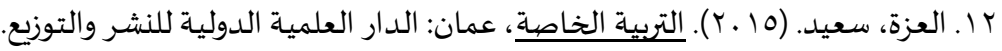

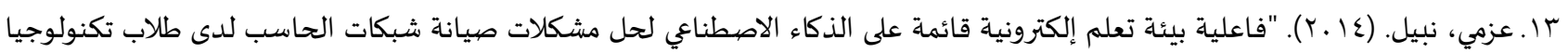

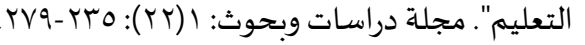

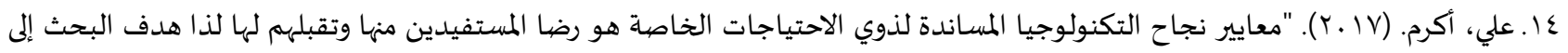
استخدام نموذج قبول التكنولوجيا TAM لتقصى فعالية التكنولوجيا المساندة القائمة على تطبيقات التعلم التكيفية النقالة لتمكين ذوي

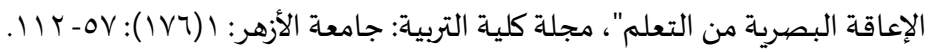
ا ـ الكحلوت، أحمد والمقيد، سامر. (V ا ـ Y). "متطلبات توظيف الذكاء الاصطناعي في العملية التعليمية في الجامعات الفلسطينية، مؤتمر التعلّم

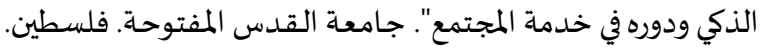

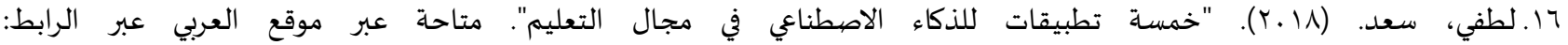
https://www.alaraby.co.uk/specialpages/2018/1/9

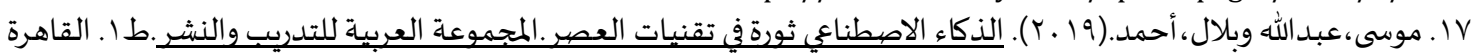
1/. النجار، محمد. (Y ا ـ Y). "فعالية برنامج قائم على تكنولوجيا الذكاء الاصطناعي في تنمية مهارات بناء المواقع الإلكترونية التعليمية لدى طلاب شعبة تكنولوجيا المعلومات في ضوء معايير الجودة الشـاملة". رسالة ماجستير. معهد الدراسات والبحوث التربوية. قسم تكنولوجيا التعليم. 


$$
\text { ثانياً: المراجع الأجنبية: }
$$

[1] Abu Hasanein, A, H (2018). “An Intelligent Tutoring System for Developing Education Case Study (Israa University)”. A Thesis Submitted for the Degree of Master. Faculty of Engineering \& Information Technology. Al-Azhar University-Gaza.

[2] Barbara, F., Armando, P., Liston, Bailey \& Belinda, M. (2018). "Perceptions of robotics emulation of human ethics in educational settings: a content analysis". Journal of Research in Innovative Teaching \& Learning: 11(2): 126-138, https://doi.org/10.1108/jrit-02-2018-0004.

[3] Barrett, M., Branson, L., Carter, S., DeLeon, F., Ellis, J., Gundlach, C., \& Lee, D. (2019). “Using Artificial Intelligence to Enhance Educational Opportunities and Student Services in Higher Education". Inquiry: The Journal of the Virginia Community Colleges.22 (1).

[4] Beal, Carole R. \& Rosenblum, L. Penny (2018). "Evaluation of the Effectiveness of a Tablet Computer Application (App) in Helping Students with Visual Impairments Solve Mathematics Problems". Journal of Visual Impairment \& Blindness. 112 (1): 5-19, https://doi.org/10.1177/0145482x1811200102.

[5] Ido R. \& Ruth W. (2016). "Evolution and Revolution in Artificial Intelligence in Education". International Artificial Intelligence in Education Society. 26(2): 582-599, https://doi.org/10.1007/s40593-016-0110-3.

[6] Makoye ،C. N. (2018). "An Investigation of Challenges Facing Students with Visual Impairment in Accessing E-Learning: A Case of Tabora Municipality”. (Doctoral dissertation ،The Open University of Tanzania).

[7] McLaughlin, R. \& Kamei-Hannan, C. (2018). "Which Reading Medium Is Best for Students with Visual Impairments?" Journal of Visual Impairment \& Blindness. 112(4): 337-350, https://doi.org/10.1177/0145482x1811200401.

[8] Monica. C.; Ali. F., Leon B. \& Paul M. (2018). "Education 4.0 - Artificial Intelligence assisted Higher Education: Early recognition System with Machine Learning to support Students' Success". IEEE 24th International Symposium for Design and Technology in Electronic Packaging (SIITME), pp: 23-30.

[9] Ocaña-Fernandez, Y., Valenzuela-Fernandez, L., \& Garro- Aburto, L. (2019). "Artificial Intelligence and its Implications in Higher Education”. Propósitos y Representations. 7(2), 536-568. http://dx.doi.org/10.20511/pyr2019.v7n2.274.

[10] OIRA ،M. (2018). "Use of modern assistive technology and its effects in educational achievement of students with visual impairment at Kibos Special Secondary School Kisumu country, Kenya”. Doctoral dissertation. Kenyatta University.

[11] Ping Mu, (2019). "Research on Artificial Intelligence Education and Its Value Orientation". 1st International Education Technology and Research Conference (IETRC 2019), pp: 771- 775.

[12] Po-Hsuan l., Andrew W., Joseph T. \&Walter m. Y. (2018). "Artificial Intelligence, the missing piece of online education?". Ieee engineering management review. 46(3): 25- 28, https://doi.org/10.1109/emr.2018.2868068.

[13] Said, S. R. (2018). "ICT Accessibility Solutions to Persons with Visual Impairment at the Open University of Tanzania”. for the degree of master of science information and communication technologies of the open university of tanzania.

[14] Woolf, B. (2013). "Building Intelligent Interactive Tutors: Student-centered Strategies for Revolutionizing E-learning". Massachusetts: Morgan Kaufmann.

[15] Zawacki-Richter Olaf, Z-R, Victoria I. Marín, Melissa B., Franziska, G. (2019). "Systematic review of research on artificial intelligence applications in higher education- where are the educators?". International Journal of Educational Technology in Higher Education. 16(1): 16-39, https://doi.org/10.1186/s41239-019-0171-0. 


$$
\text { المجلة الدولية للدراسات التربوية والنفسية }
$$

International Journal of Educational \& Psychological Studies (EPS)

Journal Homepage: https://www.refaad.com/views/EPSR/Home.aspx

www.refaad.com

ISSN: 2520-4149 (Online) 2520-4130 (Print)

\title{
The reality of using female teachers at the special education schools the to the educational applications of artificial intelligence (AI) and their attitude towards it
}

\author{
Samia Fadel Alghamdi \\ Researcher PhD in Educational Technology, King Abdul-Aziz University-Jeddah, KSA \\ samia.alghamdi@gmail.com \\ leena Ahmad Alfarani \\ Assistant Professor of Educational Technology, King Abdul-Aziz University-Jeddah, KSA
}

Received Date : $5 / 2 / 2020$

Accepted Date : $20 / 3 / 2020$

DOI : https://doi.org/DOI:10.31559/EPS2020.8.1.4

\begin{abstract}
:
This study aimed at identifying the reality of using female teachers at the special education schools the to the educational applications of artificial intelligence (AI) and their attitude towards it, from the perspective of female teachers at Al-Nur institute in Jeddah governorate. The researcher used the descriptive methodology to achieve these objectives. The sample of the study consisted of (27) female teachers at Al-Nur institute. They were chosen on purpose. The researcher prepared a questionnaire, it was consisted of (40) items divided into four fields. The results of the study showed that the importance of using educational application of AI obtained a degree (strongly agree), the obstacles of using educational application of AI and he attitudes towards using educational application of AI obtained a degree (agree), while the knowledge level and the skills related to using AI, the importance of using educational application of AI obtained a degree (neutral). The results also showed that there were no statistically significant differences at level ( $\alpha$ $=0.05$ ) according to all the variables of the study.
\end{abstract}

\section{Keywords: Special Education; Educational Applications; Artificial Intelligence.}

\section{References:}

[1] 'bd Alrazq, 'dy Wmhdy, Hydr. (2012). "Aldka' Alastna'y Wmsa'b Ttbyqh Fy Tknwlwjya Alm'lwmat". Mjlt Klyt Altrbyh Alasasyh: Bhwth Alm'etmr Alrab' Lklyh Altrbyh Alasasyh Bjam't Babl: 248- 257.

[2] 'bd Almjyd, Qtybh. (2010). "Astkhdam Aldka' Alastna'y Fy Ttbyqat Alhndsh Alkhrba'yh". Rsalt Majstyr Ghyr Mnshwrh. Aldnmark: Alakadymyh Al'rbyh.

[3] 'Ezmy, Nbyl. (2014). "Fa'lyt By'h T'lm Elktrwnyh Qa'mh 'la Aldka' Alastna'y Lhl Mshklat Syanh Shbkat Alhasb Lda Tlab Tknwlwjya Alt'lym". Mjlt Drasat Wbhwth: 1(22): 235-279.

[4] 'ly, Akrm. (2017). "M'ayyr Njah Altknwlwjya Almsandh Ldwy Alahtyajat Alkhash Hw Rda Almstfydyn Mnha Wtqblhm Lha Lda Hdf Albhth Ela Astkhdam Nmwdj Qbwl Altknwlwjya Tam Ltqsa F'alyt Altknwlwjya Almsandh Alqa'mh 'la Ttbyqat Alt'lm Altkyfyh Alnqalh Ltmkyn Dwy Ale'aqh Albsryh Mn Alt'elm", Mjlt Klyt Altrbyh: Jam't Alazhr: 1(176): 57- 112.

[5] Al'twm, N'ym Whtamlh, Habs Walsmady, 'Ely. (2019). "Dwr Altqnyat Almsandh Alkhash Balmkfwfyn Fy Tmkynhm Alajtma'y Mn Wjht Nzrhm". Mjlt Aljam't Aleslamyh Lldrasat Altrbwyh Walnfsyh: 27 (1):570-588

[6] Al'zh, S'yd. (2015). Altrbyh Alkhash, 'man: Aldar Al'Imyh Aldwlyh Llnshr Waltwzy'.

[7] Abu Hasanein, A, H (2018). “An Intelligent Tutoring System for Developing Education Case Study (Israa University)”. A Thesis Submitted for the Degree of Master. Faculty of Engineering \& Information Technology. Al-Azhar University-Gaza.

[8] Barbara, F., Armando, P., Liston, Bailey \& Belinda, M. (2018). "Perceptions of robotics emulation of human ethics in educational settings: a content analysis". Journal of Research in Innovative Teaching \& Learning: 11(2): 126-138, https://doi.org/10.1108/jrit-02-2018-0004.

[9] Barrett, M., Branson, L., Carter, S., DeLeon, F., Ellis, J., Gundlach, C., \& Lee, D. (2019). "Using Artificial Intelligence to Enhance Educational Opportunities and Student Services in Higher Education". Inquiry: The Journal of the Virginia Community Colleges.22 (1). 
[10] Beal, Carole R. \& Rosenblum, L. Penny (2018). “Evaluation of the Effectiveness of a Tablet Computer Application (App) in Helping Students with Visual Impairments Solve Mathematics Problems". Journal of Visual Impairment \& Blindness. 112 (1): 5-19, https://doi.org/10.1177/0145482x1811200102.

[11] Ido R. \& Ruth W. (2016). "Evolution and Revolution in Artificial Intelligence in Education". International Artificial Intelligence in Education Society. 26(2): 582-599, https://doi.org/10.1007/s40593-016-0110-3.

[12] Jad Allh, Ahmd (2019). "Fa'lyt Brnamj Tdryby Qa'm 'la Ttbyqat Jwjl Alt'lymyh Fy Tnmyh Mharat Alt'lm Alelktrwny Lda Alm'lmyn Dwy Ale'aqh Albsryh". RsaltMajstyr. Klyt Altrbyh: Mnahj Wtrq Tdrys. Aljam'h Aleslamyh. Ghzh/ Flstyn.

[13] Alkhlwt, Ahmd Walmqyd, Samr. (2017). "Mttlbat Twzyf Aldka' Alastna'y Fy Al'mlyh Alt'lymyh Fy Aljam'at Alflstynyh, M'tmr Alt'lm Aldky Wdwrh Fy Khdmt Almjtm'". Jam't Alqds Almftwhh. Flstyn.

[14] Alkhtyb, Jmal Walhdydy, Mna. (2011). Almdkhl Ela Altrbyh Alkhash. 'man: Mtb't Dar Alfkr.

[15] Ltfy, S'd. (2018). "Khmsh Ttbyqat Lldka' Alastna'y Fy Mjal Alt'lym". Mtahh 'br Mwq' Al'rby 'br Alrabt: https://www.alaraby.co.uk/specialpages/2018/1/9

[16] Makoye ،C. N. (2018). "An Investigation of Challenges Facing Students with Visual Impairment in Accessing E-Learning: A Case of Tabora Municipality". (Doctoral dissertation ،The Open University of Tanzania)

[17] McLaughlin, R. \& Kamei-Hannan, C. (2018). “Which Reading Medium Is Best for Students with Visual Impairments?” Journal of Visual Impairment \& Blindness. 112(4): 337-350, https://doi.org/10.1177/0145482x1811200401.

[18] Monica. C.; Ali. F., Leon B. \& Paul M. (2018). "Education 4.0 - Artificial Intelligence assisted Higher Education: Early recognition System with Machine Learning to support Students' Success". IEEE 24th International Symposium for Design and Technology in Electronic Packaging (SIITME), pp: 23-30.

[19] Mwsa,'bdallh Wblal, Ahmd. (2019). Aldka' Alastna'y Thwrh Fy Tqnyat Al'sr.Almjmw'h Al'rbyh Lltdryb Walnshr.T1. Alqahrh

[20] Alnjar, Mhmd. (2012). "F'alyt Brnamj Qa'm 'la Tknwlwjya Aldka' Alastna'y Fy Tnmyt Mharat Bna' Almwaq' Alelktrwnyh Alt'lymyh Lda Tlab Sh'bh Tknwlwjya Alm'lwmat Fy Dw' M'ayyr Aljwdh Alshamlh". Rsalt Majstyr. M'hd Aldrasat Walbhwth Altrbwyh. Qsm Tknwlwjya Alt'lym.

[21] Ocaña-Fernandez, Y., Valenzuela-Fernandez, L., \& Garro- Aburto, L. (2019). "Artificial Intelligence and its Implications in Higher Education". Propósitos y Representations. 7(2), 536-568. http://dx.doi.org/10.20511/pyr2019.v7n2.274.

[22] OIRA ،M. (2018). "Use of modern assistive technology and its effects in educational achievement of students with visual impairment at Kibos Special Secondary School Kisumu country, Kenya”. Doctoral dissertation. Kenyatta University.

[23] Ping Mu, (2019). "Research on Artificial Intelligence Education and Its Value Orientation". 1st International Education Technology and Research Conference (IETRC 2019), pp: 771- 775.

[24] Po-Hsuan l., Andrew W., Joseph T. \&Walter m. Y. (2018). "Artificial Intelligence, the missing piece of online education?". Ieee engineering management review. 46(3): 25- 28, https://doi.org/10.1109/emr.2018.2868068.

[25] S'd Allh, 'mar Wshtwh, Wlyd. (2019). "Ahmyh Aldka' Alastna'y Fy Ttwyr Alt'lym. Ttbyqat Aldka' Alastna'y Ktwjh Hdyth Lt'ezyz Tnafsyh Mnzmat Ala'mal", Brlyn: Almrkz Aldymqraty Al'rby.

[26] Al S'wd, Sarh. (2016). "Alttbyqat Altrbwyh Lldka' Alastna'y Fy Aldrasat Alajtma'yh". Mjlh Slwk: 3(4): 133-163.

[27] Said, S. R. (2018). "ICT Accessibility Solutions to Persons with Visual Impairment at the Open University of Tanzania". for the degree of master of science information and communication technologies of the open university of tanzania.

[28] Shhath, Hazm. (2018). "Astkhdam Altqnyat Almsa'dh Fy Aljam'at Alflstynyh Wathrha 'la Altlab Dwy Ale'aqh Albsryh: Drash Halt Aljam'h Aleslamyh Bghzh". Mjlt Aldrasat Al'lya: 11(44): 187-204.

[29] Abw Shmalh, Rsha. (2013). "Fa'lyt Brnamj Qa'm 'la Aldka' Alastna'y Ltnmyh Altfkyr Alastdlaly Walthsyl Aldrasy Fy Mbhth Tknwlwjya Alm'lwmat Lda Talbat Alhady 'shr Bghzh". Rsalt Majstyr Bklyt Altrbyh Jam't Alazhr. Ghzh.

[30] Alsyd, Hwyda. (2014). "Fa'lyt Brnamj Tdryby Elktrwny Fy Eksab B'd Mharat Astkhdam Mwaq' Altwasl Alajtma'y Walthqh Balnfs Walatjah Nhw Astkhdamha Lda Almkfwfyn". Mjlt Klyt Altrbyh, Jam't Alazhr - Klyt Altrbyh: 349- 393.

[31] Tlbh, Rham. (2018). "Astkhdam Albrmjyat Alt'lymyh Mftwhh Almsdr Oer Fy Tnmyt Alkfayat Alada'yh Alelktrwnyh Lltlab Dwy Ale'aqh Albsryh Fy Mdrsh Alnwr Bmhafzh Asywt". Mjlh Al'lwm Altrbwyh Walnfsyh, Almjlh Al'rbyh Ll'lwm Wnshr Alabhath: 2 (10): 1-25.

[32] Woolf, B. (2013). "Building Intelligent Interactive Tutors: Student-centered Strategies for Revolutionizing E-learning". Massachusetts: Morgan Kaufmann.

[33] Zawacki-Richter Olaf, Z-R, Victoria I. Marín, Melissa B., Franziska, G. (2019). "Systematic review of research on artificial intelligence applications in higher education- where are the educators?". International Journal of Educational Technology in Higher Education. 16(1): 16-39, https://doi.org/10.1186/s41239-019-0171-0. 